\title{
IRREDUCIBLE MODULE HOMOMORPHISMS OF A VON NEUMANN ALGEBRA INTO ITS CENTER $\left({ }^{1}\right)$
}

\author{
BY \\ HERBERT HALPERN
}

1. Introduction. A von Neumann algebra $\mathscr{A}$ can be considered as a module over its center $\mathscr{Z}$. The norm of $\mathscr{A}$ induces a norm on the module $\mathscr{A}$. Whenever we talk of the module $\mathscr{A}$ it will always be this specific module over $\mathscr{Z}$. In this article we study the set $\mathscr{A}^{\sim}$ of bounded module homomorphisms of $\mathscr{A}$ into $\mathscr{Z}$. In an earlier article we studied those module homomorphisms of $\mathscr{A}$ into $\mathscr{Z}$ which are continuous in the $\sigma$-weak topology of $\mathscr{A}$ and $\mathscr{Z}$ respectively. In that paper we discovered a specific form for such homomorphisms and showed that a type I algebra could be characterized in terms of such functionals. These results were analogues of results known for factor algebras. For factor algebras multipliers are scalars and the mappings are scalar-valued functionals while in algebras with arbitrary centers the multipliers are central elements and the mappings are module homomorphisms into the center.

There are always module homomorphisms of $\mathscr{A}$ into $\mathscr{Z}$. A kind which is particularly simple although fundamental may be constructed as follows. Let $\mathscr{Z}^{\prime}$ be the commutator of $\mathscr{Z}$ and let $E$ be an abelian projection in $\mathscr{Z}^{\prime}$ with central support $P$. There is an isomorphism of $\mathscr{Z} P$ onto $E \mathscr{Z}{ }^{\prime} E$ given by $A \rightarrow A E$. For each $A$ in $\mathscr{A}$ we denote the inverse image in $\mathscr{Z} P$ of $E A E$ under this isomorphism by $\tau_{E}(A)$. Then the function $\tau_{E}$ on $\mathscr{A}$ is a homomorphism into $\mathscr{Z}$.

In general $\mathscr{Z}$ is a most suitable range for module homomorphisms. The following Hahn-Banach type theorem illustrates this. Let $\mathscr{B}$ be a normed space which is a module over a commutative $A W^{*}$-algebra $\mathscr{Z}$. Let $\mathscr{C}$ be any submodule of $\mathscr{B}$ and let $\phi$ be a bounded module homomorphism of $\mathscr{C}$ into $\mathscr{Z}$. There is a bounded module homomorphism $\psi$ of $\mathscr{B}$ into $\mathscr{Z}$ such that $\psi(C)=\phi(C)$ for every $C$ in $\mathscr{C}$ and such that $\|\psi\|=\|\phi\|[19],[24]$. From this theorem many homomorphisms may be constructed.

A module homomorphism $\phi$ of $\mathscr{A}$ into $\mathscr{Z}$ will be called a functional of the module $\mathscr{A}$. A functional $\phi$ of the module $\mathscr{A}$ is said to be hermitian if $\phi\left(A^{*}\right)=\phi(A)^{*}$ for every $A$ in $\mathscr{A}$. Every bounded functional of the module $A$ can be written as a linear combination of two bounded hermitian functionals. A functional $\phi$ of the module $\mathscr{A}$ is said to be positive if $\phi$ maps $\mathscr{A}^{+}$into $\mathscr{Z}^{+}$. Since

$$
|\phi(A)|^{2}=\phi(A)^{*} \phi(A) \leqq \phi\left(A^{*} A\right) \phi(1),
$$

every positive functional $\phi$ is bounded with bound $\|\phi(1)\|$. Every bounded hermitian

Received by the editors June 10, 1968.

( ${ }^{1}$ The research for this paper was partially supported by the National Science Foundation. 
functional of the module $\mathscr{A}$ may be written as the difference of two positive functionals of the module $\mathscr{A}$ [19], [24].

In this paper we study the positive functionals of the module $\mathscr{A}$. The set $\mathscr{S}$ of positive functionals of $\mathscr{A}^{\sim}$ of norm not exceeding 1 is compact in a naturally defined topology in $\mathscr{A} \sim$. The set $\mathscr{S}$ has extreme points and $\mathscr{S}$ is the closure (in this topology) of the convex hull of its extreme points. Here though the convexity is expressed in terms of multiplication by elements in $\mathscr{Z}$. We show that every linear functional $f$ on $\mathscr{A}$ which is $\sigma$-weakly continuous when restricted to $\mathscr{Z}$ can be expressed as the composition of $f$ with an element of $\mathscr{A}^{\sim}$.

A positive functional $\phi$ in $\mathscr{A}^{\sim}$ normalized so that $\phi(1)=1$ gives rise to a representation of $\mathscr{A}$ as a *-subalgebra of the algebra of all bounded linear operators on an $A W^{*}$-module $M_{\phi}$ over the center $\mathscr{Z}$ ([6], [17], [28]). We study the representations that arise from an extreme point $\phi$ of $\mathscr{S}$. By presenting a specific form for the representation we are able to obtain the analogue of Kadison's theorem on strict irreducibility. If $A \rightarrow A^{\wedge}$ denotes the Gelfand transform of $\mathscr{Z}$ onto the algebra of all continuous complex-valued functions on the spectrum $Z$ of $\mathscr{Z}$, then the analogue of Kadison's theorem allows us to conclude that $A \rightarrow \phi(A)^{\wedge}(\zeta)$ is a pure state of $\mathscr{A}$ for every $\zeta$ in $\mathscr{Z}$. In a certain sense this result illustrates the advantage of a global theory over a decomposition theory. By an additional construction we are able to find an extreme point $\phi$ such that the kernel of the canonical representation of $\mathscr{A}$ on a Hilbert space induced by $A \rightarrow \phi(A)^{\wedge}(\zeta)$ ( $\zeta$ fixed but arbitrary in $Z$ ) is the

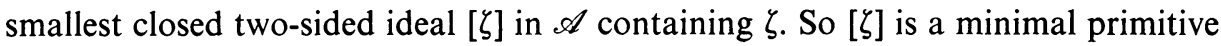
ideal of $\mathscr{A}$.

We then define a vector state of the $\mathscr{A}$ as a module. This definition comes from ideas in a previous paper [12]. The set of elements in $\mathscr{A}^{\sim}$ obtained as pointwise limits of these vector states is called the vector state space. The set of pointwise limits in $\mathscr{A}^{\sim}$ of the set of extreme points $\phi$ of the positive elements of the unit sphere of $\mathscr{A}^{\sim}$ which satisfy $\phi(1)=1$ is called the pure state space of the module $\mathscr{A}$. We then compare the set of all $\phi$ in the unit sphere of $\mathscr{A} \sim$ such that $\phi(1)=1$ with the pure state space and the vector state space of the module $\mathscr{A}$. These structures have exactly the same relations as the corresponding structures of scalar functionals as given by Glimm ([3], [4]). Here the ideal of completely continuous operators is replaced by the ideal generated by the abelian projections of $\mathscr{A}$.

2. Existence of extreme points. Let $\mathscr{A}$ be a von Neumann algebra with center $\mathscr{Z}$ and let $\mathscr{A} \sim$ be the space of bounded functionals of the module $\mathscr{A}$. Let $\mathscr{Z}_{*}$ be the set of all $\sigma$-weakly continuous functionals on $\mathscr{Z}$. For each $f$ in $\mathscr{Z}_{*}$ and $A$ in $\mathscr{A}$ define the seminorm $p_{f, A}=p$ of $\mathscr{A}^{\sim}$ by $p(\phi)=|f(\phi(A))|$. The family $\left\{p_{f, A} \mid f \in \mathscr{Z}_{*}, A \in \mathscr{A}\right\}$ of seminorms of $\mathscr{A}^{\sim}$ defines a topology on $\mathscr{A}^{\sim}$ under which $\mathscr{A}^{\sim}$ is a locally convex Hausdorff topological linear space. We call this topology the weak-* topology of $\mathscr{A}^{\sim}$. If $f$ is a weak-* continuous functional on $\mathscr{A}^{\sim}$, there are functionals $f_{1}, f_{2}, \ldots, f_{n}$ in $\mathscr{Z}_{*}$ and $A_{1}, A_{2}, \ldots, A_{n}$ in $\mathscr{A}$ such that 


$$
f(\psi)=\sum\left\{f_{j}\left(\psi\left(A_{j}\right)\right) \mid 1 \leqq j \leqq n\right\}
$$

for every $\psi \in \mathscr{A}^{\sim}$. Since every positive functional $g$ in $\mathscr{Z}_{*}$ is of the form $g(A)$ $=(A x, x)$ for some vector $x$ of the Hilbert space $H$ of $\mathscr{Z}$, we have that there are vectors $x_{1}, x_{2}, \ldots, x_{m}, y_{1}, y_{2}, \ldots, y_{m}$ in $H$ and $B_{1}, B_{2}, \ldots, B_{m}$ in $\mathscr{Z}$ such that

$$
f(\psi)=\sum\left\{\left(\psi\left(B_{j}\right) x_{j}, y_{j}\right) \mid 1 \leqq j \leqq m\right\} .
$$

Proposition 2.1. Let $\mathscr{A}$ be a von Neumann algebra. Let $\mathscr{A}_{\tilde{1}}$ be the unit sphere of the set $\mathscr{A} \sim$ of bounded functionals of the module $\mathscr{A}$ and let $\mathscr{S}$ be the set of positive elements of $\mathscr{A}_{1}$. The sets $\mathscr{A}_{1}$ and $\mathscr{S}$ are compact in the weak-* topology of $\mathscr{A}^{\sim}$.

Proof. Let $\mathscr{Z}_{A}=\mathscr{Z}$ for every $A \in \mathscr{A}$. Let $\prod\left\{\mathscr{Z}_{A} \mid A \in \mathscr{A}\right\}$ be the product space of $\left\{\mathscr{Z}_{A} \mid A \in \mathscr{A}\right\}$ supplied with the product topology induced by the $\sigma$-weak topology on each $\mathscr{Z}_{A}$. Let $\Phi$ be a function of $\mathscr{A}^{\sim}$ into $\prod \mathscr{Z}_{A}$ given by $\Phi(\phi)_{A}=\phi(A)$. The function $\Phi$ is an isomorphism of $\mathscr{A}^{\sim}$ onto $\Phi\left(\mathscr{A}^{\sim}\right)$ which is bicontinuous when $\mathscr{A}^{\sim}$ is supplied with the weak-* topology. Let $\mathscr{N}=\prod\left\{\mathscr{N}_{A} \mid A \in \mathscr{A}\right\}$ be the subset of $\Pi \mathscr{Z}_{A}$ defined by the relation

$$
\mathscr{N}_{A}=\left\{B \in \mathscr{Z}_{A} \mid\|B\| \leqq\|A\|\right\} .
$$

The set $\mathscr{N}$ is compact in $\prod \mathscr{Z}_{A}$. Since $\left\|\Phi(\phi)_{A}\right\| \leqq\|A\|$ whenever $\phi \in \mathscr{A}_{1}$, it is sufficient to show that $\Phi\left(\mathscr{A}_{1}^{\tilde{1}}\right)$ is closed in $\mathscr{N}$ in order to show $\mathscr{A}_{1}$ is compact in the weak-* topology. Let $\left\{\psi_{n}\right\}$ be a net in $\mathscr{A}_{i}$ such that $\left\{\Phi\left(\psi_{n}\right)\right\}$ converges to an element $\rho$ in $\mathscr{N}$. Let $f$ be an element of $\mathscr{Z}_{*}, A_{1}$ and $A_{2}$ be elements of $\mathscr{A}$, and $C_{1}$ and $C_{2}$ be elements of $\mathscr{Z}$. Since the nets

$$
\left\{f\left(\psi_{n}\left(C_{1} A_{1}\right)\right)\right\}, \quad\left\{f\left(\psi_{n}\left(C_{2} A_{2}\right)\right)\right\} \text { and }\left\{f\left(\psi_{n}\left(C_{1} A_{1}+C_{2} A_{2}\right)\right)\right\}
$$

converge to

$$
f\left(C_{1} \rho_{A_{1}}\right), \quad f\left(C_{2} \rho_{A_{2}}\right) \text { and } f\left(\rho_{\left(C_{1} A_{1}+C_{2} A_{2}\right)}\right)
$$

respectively, we have that

$$
f\left(C_{1} \rho_{A_{1}}+C_{2} \rho_{A_{2}}\right)=f\left(\rho_{\left(C_{1} A_{1}+C_{2} A_{2}\right)}\right) .
$$

Because $f$ is arbitrary, we have that

$$
C_{1} \rho_{A_{1}}+C_{2} \rho_{A_{2}}=\rho_{\left(C_{1} A_{1}+C_{2} A_{2}\right)} \text {. }
$$

Therefore, the function $A \rightarrow \rho_{A}$ is a module homomorphism $\phi$ of $\mathscr{A}$ into $\mathscr{Z}$. But $\|\phi(A)\| \leqq\|A\|$ and therefore $\phi$ is an element of $\mathscr{A}_{\tilde{1}}$. This proves $\Phi\left(\mathscr{A}_{1}\right)$ is closed in $\mathscr{N}$.

Now we show that $\mathscr{S}$ is weak-* compact in $\mathscr{A}^{\sim}$. Let $\left\{\psi_{n}\right\}$ be a net in $\mathscr{S}$ converging in the weak-* topology to a point $\psi$ in $\mathscr{A}_{1}$. But if $A$ is a positive element of $\mathscr{A}$, then

$$
f(\psi(A))=\lim _{n} f\left(\psi_{n}(A)\right) \geqq \lim \inf f\left(\psi_{n}(A)\right) \geqq 0
$$

for every positive $\sigma$-weakly continuous $f$ functional of $\mathscr{Z}$. Thus $\psi(A) \geqq 0$ for every 
$A \geqq 0$. This proves that $\mathscr{S}$ is closed in $\mathscr{A}_{1}^{\tilde{1}}$. So $\mathscr{S}$ is compact in the weak-* topology. Q.E.D.

Let $\mathscr{A}$ be a von Neumann algebra with center $\mathscr{Z}$. The space $\mathscr{A} \sim$ of bounded functionals on the module $\mathscr{A}$ is a locally convex linear topological space with the weak-* topology. A linear functional $f$ on $\mathscr{A} \sim$ is said to be hermitian if $f(\phi)$ is real for every hermitian functional $\phi$ in $\mathscr{A} \sim$. If $\mathscr{K}$ is a nonvoid convex weak-* closed subset of $\mathscr{A}^{\sim}$ and if $\phi$ is an element of the complement of $\mathscr{K}$, there is a weak-* continuous functional $f$ of $\mathscr{A}^{\sim}$ such that

$$
\operatorname{lub}\{\operatorname{Re} f(\psi) \mid \psi \in \mathscr{K}\}<\operatorname{Re} f(\phi) .
$$

Here $\operatorname{Re} \alpha$ denotes the real part of the number $\alpha$. Suppose $\phi$ is hermitian and the elements of $\mathscr{K}$ are hermitian. Let $f(\psi)=\sum_{j}\left(\psi\left(A_{j}\right) x_{j}, y_{j}\right)$ where $x_{1}, x_{2}, \ldots, x_{n}$, $y_{1}, y_{2}, \ldots, y_{n}$ are vectors of the Hilbert space of $\mathscr{A}$ and $A_{1}, A_{2}, \ldots, A_{n}$ are elements of $\mathscr{A}$. Let $g(\psi)=\sum\left(\psi\left(A_{j}^{*}\right) y_{j}, x_{j}\right)$. The functional $h(\psi)=(f(\psi)+g(\psi)) / 2$ is a weak-* continuous hermitian functional on $\mathscr{A}^{\sim}$ which coincides with $\operatorname{Re} f$ on $\mathscr{K} \cup\{\phi\}$. This means that there is a weak-* continuous hermitian functional $h$ of $\mathscr{A}^{\sim}$ such that

$$
\operatorname{lub}\{h(\psi) \mid \psi \in \mathscr{K}\}<h(\phi) .
$$

Let $\mathscr{Z}$ be a commutative von Neumann algebra and let $Z$ be the spectrum of $\mathscr{Z}$. If $C$ is an element of $\mathscr{Z}$ whose Gelfand transform $C^{\wedge}$ on $Z$ has range contained in the open interval $(0,1)$, then $C$ is said to lie strictly between 0 and 1 . If $C$ lies strictly between 0 and 1 we write $0<C<1$. If $M$ is a $\mathscr{Z}$-module, a subset $\mathscr{K}$ of $M$ will be called $\mathscr{Z}$-convex if $C A+(1-C) B$ is in $\mathscr{K}$ whenever $A$ and $B$ are in $\mathscr{K}$ and $C$ is in $\mathscr{Z}$ with $0 \leqq C \leqq 1$. A point $A$ of a $\mathscr{Z}$-convex subset $\mathscr{K}$ of $M$ is said to be an extreme point of $\mathscr{K}$ if $C B+(1-C) D=A$ implies $B=D=A$ whenever $B$ and $D$ are elements of $\mathscr{K}$ and $C$ is an element of $\mathscr{Z}$ strictly between 0 and 1.

TheOREM 2.2. Let $\mathscr{A}$ be a von Neumann algebra with center $\mathscr{Z}$ and let $\mathscr{S}$ be the set of positive functionals of norm not exceeding 1 of the module $\mathscr{A}$. If $\mathscr{K}$ is a nonvoid $\mathscr{Z}$-convex weak-* compact subset of $\mathscr{S}$, then $\mathscr{K}$ is the weak-* closure of the smallest $\mathscr{Z}$-convex subset of $\mathscr{K}$ containing the extreme points of $\mathscr{K}$.

Proof. Let $B$ be an element of $\mathscr{A}^{+}$. The set $\{\phi(B) \mid \phi \in \mathscr{K}\}$ is a monotonely increasing net in $\mathscr{Z}^{+}$which is bounded above. Let $B_{0}=\operatorname{lub}\{\phi(B) \mid \phi \in \mathscr{K}\}$. The $\mathscr{Z}$-convex set $S(B)=\left\{\phi \in \mathscr{K} \mid \phi(B)=B_{0}\right\}$ is nonvoid and contains an extreme point of $\mathscr{K}$. This was demonstrated in Theorem 7 [12] for an analogous situation and virtually the same demonstration applies here.

Let $\mathscr{K}^{\prime}$ be the weak-* closure of the smallest $\mathscr{Z}$-convex subset of $\mathscr{K}$ containing the set of extreme points of $\mathscr{K}$. We show that $\mathscr{K}^{\prime}=\mathscr{K}$ by arguing by contradiction. Suppose there is an element $\phi$ in the complement of $\mathscr{K}^{\prime}$ with respect to $\mathscr{K}$. There is a weak-* continuous hermitian functional $f$ of $\mathscr{A}^{\sim}$ such that

$$
\operatorname{lub}\left\{f(\psi) \mid \psi \in \mathscr{K}^{\prime}\right\}<f(\phi)
$$


Let

$$
T=\{\theta \in \mathscr{K} \mid f(\theta)=\operatorname{lub}\{f(\psi) \mid \psi \in \mathscr{K}\}\} .
$$

Since $\mathscr{K}$ is a weak-* compact set and since $f$ is weak-* continuous, the set $T$ is a nonvoid weak-* compact subset of $\mathscr{K}$. We show that $T$ is $\mathscr{Z}$-convex. Let $P$ be a projection in $\mathscr{Z}$. We have that

$$
(\psi(A) x, y)=(P \psi(A) x, y)+((1-P) \psi(A) x, y)
$$

for every $\psi \in \mathscr{A}^{\sim}, A \in \mathscr{A}$, and $x$ and $y$ in the Hilbert space of $\mathscr{A}$. Thus $f(\psi)=f(P \psi)$ $+f((1-P) \psi)$ for every $\psi$ in $\mathscr{A}^{\sim}$. Now let $\theta$ be an element of $T$. We have that $f(P \theta)=\operatorname{lub}\{f(P \psi) \mid \psi \in \mathscr{K}\}$. Indeed, if there is a $\psi$ in $\mathscr{K}$ with $f(P \theta)<f(P \psi)$ we have that

$$
f(P \psi+(1-P) \theta)=f(P \psi)+f((1-P) \theta)>f(P \theta)+f((1-P) \theta)=f(\theta) .
$$

However, the function at $P \psi+(1-P) \theta$ is an element of $\mathscr{K}$. We have reached a contradiction. So,

$$
f(P \theta)=\operatorname{lub}\{f(P \psi) \mid \psi \in \mathscr{K}\} .
$$

This means that $f(P \theta)=f(P \psi)$ for any two elements $\theta$ and $\psi$ in $T$ and any central projection $P$. Now let $C$ be any element in $\mathscr{Z}^{+}$. Let $\varepsilon>0$ be given; let $\left\{P_{j} \mid 1 \leqq j \leqq n\right\}$ be mutually orthogonal projections of $\mathscr{Z}$ and let $\left\{\alpha_{j} \mid 1 \leqq j \leqq m\right\}$ be nonnegative scalars such that $\left\|C-\sum \alpha_{j} P_{j}\right\|<\varepsilon$. If $\theta$ and $\psi$ are elements of $T$, then

$$
|f(C \theta)-f(C \psi)| \leqq\left|f(C \theta)-f\left(\left(\sum \alpha_{j} P_{j}\right) \theta\right)\right|+\left|f\left(\left(\sum \alpha_{j} P_{j}\right) \psi\right)-f(C \psi)\right| \leqq 2 \varepsilon\|f\| .
$$

Since $\varepsilon$ is arbitrary, we see that $f(C \theta)=f(C \psi)$. Thus the set $T$ is $\mathscr{Z}$-convex. Now by the remarks made at the beginning of this proof we can conclude that $T$ has an extreme point $\phi_{0}$. We show that $\phi_{0}$ is an extreme point of $\mathscr{K}$. Indeed, let $\phi_{1}$ and $\phi_{2}$ be elements of $\mathscr{K}$ such that $C \phi_{1}+(1-C) \phi_{2}=\phi_{0}$ for some central element $C$ strictly between 0 and 1 . Let $D$ be a positive central element; let $\varepsilon>0$ be given and let $\left\{P_{j} \mid 1 \leqq j \leqq n\right\}$ be mutually orthogonal central projections such that $\left\|D-\sum \alpha_{j} P_{j}\right\|$ $\leqq \varepsilon$ for suitable nonnegative scalars $\left\{\alpha_{j} \mid 1 \leqq j \leqq n\right\}$. Because

$$
f\left(P_{j} \phi_{0}\right)=\operatorname{lub}\left\{f\left(P_{j} \theta\right) \mid \theta \in \mathscr{K}\right\} \quad \text { for } j=1,2, \ldots, n
$$

we have that

$$
f\left(\left(\sum \alpha_{j} P_{j}\right) \phi_{1}\right)=\sum \alpha_{j} f\left(P_{j} \phi_{1}\right) \leqq \sum \alpha_{j} f\left(P_{j} \phi_{0}\right)=f\left(\left(\sum \alpha_{j} P_{j}\right) \phi_{0}\right) .
$$

So we have that

$$
f\left(D \phi_{1}\right) \leqq f\left(\left(\sum \alpha_{j} P_{j}\right) \phi_{1}\right)+\varepsilon\|f\| \leqq f\left(\left(\sum \alpha_{j} P_{j}\right) \phi_{0}\right)+\varepsilon\|f\| \leqq f\left(D \phi_{0}\right)+2 \varepsilon\|f\| .
$$

Since $\varepsilon>0$ is arbitrary, we have that $f\left(D \phi_{1}\right) \leqq f\left(D \phi_{0}\right)$. So for every central projection $Q$ we may conclude that

$$
f\left(C Q \phi_{0}\right)=f\left(C Q \phi_{1}\right) \text { and } f\left((1-C) Q \phi_{0}\right)=f\left((1-C) Q \phi_{2}\right),
$$


since the sum of the two positive numbers

$$
f\left(C Q \phi_{0}\right)-f\left(C Q \phi_{1}\right) \text { and } f\left((1-C) Q \phi_{0}\right)-f\left((1-C) Q \phi_{2}\right)
$$

is zero. The elements $C$ and $1-C$ are invertible in $\mathscr{Z}^{+}$. Given $\varepsilon>0$, there are mutually orthogonal central projections $\left\{Q_{j} \mid 1 \leqq j \leqq n\right\}$ and nonnegative numbers $\left\{\alpha_{j} \mid 1 \leqq j \leqq n\right\}$ such that $\left\|C^{-1}-\sum \alpha_{j} Q_{j}\right\| \leqq \varepsilon$. Therefore,

$$
\begin{aligned}
\left|f\left(\phi_{1}\right)-f\left(\phi_{0}\right)\right| & \leqq\left|f\left(\left(1-\left(\sum \alpha_{j} Q_{j}\right) C\right) \phi_{1}\right)\right|+\left|f\left(\left(\left(\sum \alpha_{j} Q_{j}\right) C-1\right) \phi_{0}\right)\right| \\
& \leqq 2\|f\|\left\|1-\left(\sum \alpha_{j} Q_{j}\right) C\right\| \\
& \leqq 2\|f\|\|C\|\left\|C^{-1}-\sum \alpha_{j} Q_{j}\right\| \leqq 2\|f\|\|C\| \varepsilon .
\end{aligned}
$$

Since $\varepsilon>0$ is arbitrary, we have that $f\left(\phi_{1}\right)=f\left(\phi_{0}\right)$. Similarly we find that $f\left(\phi_{2}\right)$ $=f\left(\phi_{0}\right)$. This proves that both $\phi_{1}$ and $\phi_{2}$ are elements of $T$. Because $\phi_{0}$ is an extreme point of $T$, the element $\phi_{0}$ is equal to $\phi_{1}$ and $\phi_{2}$. Hence $\phi_{0}$ is an extreme point of $\mathscr{K}$. However, $\phi_{0}$ cannot be in the set $\mathscr{K}^{\prime}$. This is a contradiction. Therefore, we must have that $\mathscr{K}=\mathscr{K}^{\prime}$. Q.E.D.

In the final section of this paper we shall present some facts about the closure of the smallest $\mathscr{Z}$-convex subset of $\mathscr{S}$ containing the extreme points of $\mathscr{S}$ in the topology of pointwise convergence on $\mathscr{S}$ where $\mathscr{Z}$ is taken with the uniform topology.

Let $\mathscr{A}$ be a von Neumann algebra with center $\mathscr{Z}$. A positive functional $\phi$ of the module $\mathscr{A}$ is said to majorize a positive functional $\psi$ if $\phi-\psi$ is a positive functional of the module $\mathscr{A}$. If $\phi$ majorizes $\psi$, we write $\phi \geqq \psi$. A positive functional $\phi$ is said to be $\mathscr{Z}$-irreducible if given any positive functional $\psi$ majorized by $\phi$ then there is a positive element $C$ in $\mathscr{Z}$ such that $C \phi=\psi$. In [12] we proved the following theorem:

Let $\mathscr{A}$ be a von Neumann algebra with center $\mathscr{Z}$. Let $\mathscr{S}$ be the set of all positive functionals of the module $\mathscr{A}$ with norm not exceeding 1 . Let $\phi \in \mathscr{S}$. The following are equivalent:

(1) $\phi$ is an extreme point of $\mathscr{S}$; and

(2) $\phi(1)$ is a projection and $\phi$ is $\mathscr{Z}$-irreducible.

3. Functionals $\sigma$-weakly continuous on the center. In this section we examine the positive functionals of a von Neumann algebra which are $\sigma$-weakly continuous when restricted to the center.

If $f$ is a positive functional on a $C^{*}$-algebra $\mathscr{A}$, let $L_{f}$ be the closed left-ideal of $\mathscr{A}$ given by

$$
L_{f}=\left\{A \in \mathscr{A} \mid f\left(A^{*} A\right)=0\right\} .
$$

The space $\mathscr{A}-L_{f}$ is a prehilbert space with the inner product

$$
\left(A-L_{f}, B-L_{f}\right)=f\left(B^{*} A\right) .
$$

Let $H(f)$ be the completion of $\mathscr{A}-L_{f}$. The representation $\pi$ of $\mathscr{A}$ on $H(f)$ which extends the left multiplication of $\mathscr{A}$ on $\mathscr{A}-L_{f}$ is called the canonical representation 
of $\mathscr{A}$ induced by $f$. There is a vector $x$ in $H(f)$ which is cyclic under $\pi(\mathscr{A})$ such that $(\pi(A) x, x)=f(A)$ for every $A$ in $\mathscr{A}$.

THEOREM 3.1. Let $f$ be a positive functional of a von Neumann algebra $\mathscr{A}$. Suppose that the restriction $g$ of $f$ to the center $\mathscr{Z}$ of $\mathscr{A}$ is $\sigma$-weakly continuous. There is a unique positive functional $\phi$ of module $\mathscr{A}$ such that $f=g \cdot \phi$ and such that $\phi(1)$ is equal to the support of $g$.

Proof. Let $P$ be the support of $g$. Let $\pi$ be the canonical representation of $\mathscr{A}$ on a Hilbert space $H$ induced by $f$. Let $x$ be an element of $H$ cyclic under $\pi(\mathscr{A})$ such that $f(A)=(\pi(A) x, x)$ for every $A$ in $\mathscr{A}$. The representation $\pi$ restricted to $\mathscr{Z}$ is $\sigma$-weakly continuous. Indeed, let $\left\{A_{n}\right\}$ be a monotonely increasing net in $\mathscr{Z}^{+}$with least upper bound $A$. Then $\left\{\left(A_{n}-A\right)^{*}\left(A_{n}-A\right)\right\}$ converges $\sigma$-weakly to 0 . So

$$
\left\{g\left(\left(A_{n}-A\right)^{*}\left(A_{n}-A\right)\right)\right\}
$$

converges to 0 . This means that $\lim \pi\left(A_{n}-A\right) x=0$. Therefore, $\lim \pi\left(A_{n}\right) B x=\pi(A) B x$ for every $B \in \pi(\mathscr{A})$. Since the net $\left\{\pi\left(A_{n}\right)\right\}$ is bounded, the net $\left\{\pi\left(A_{n}\right)\right\}$ converges strongly to $\pi(A)$. This proves $\pi$ is $\sigma$-weakly continuous on $\mathscr{Z}$. This shows that $\pi(\mathscr{Z})$ is a von Neumann algebra on $H$ [1, Chapter I, $\S 3$, Theorem 2, Corollary 2].

The algebra $\mathscr{Z} P$ is isomorphic to $\pi(\mathscr{Z})$ under the map $\pi$. Let $\pi^{-1}$ denote the inverse of this map. Now let $E$ be the abelian projection of the commutator $\pi(\mathscr{Z})^{\prime}$ of $\pi(\mathscr{Z})$ on $H$ corresponding to the subspace

$$
\text { closure }\{A x \mid A \in \pi(\mathscr{Z})\} \text {. }
$$

We have that

$$
f(A)=(\pi(A) x, x)=\left(\tau_{E}(\pi(A)) x, x\right)
$$

for every $A$ in $\mathscr{A}$. Then define $\phi(A)=\pi^{-1}\left(\tau_{E}(\pi(A))\right)$. We have that $\phi$ is a positive functional of the module $\mathscr{A}$ such that $\phi(1)=P$. Also we see that

$$
g(\phi(A))=(\pi(\phi(A)) x, x)=(\pi(A) x, x)=f(A)
$$

for every $A$ in $\mathscr{A}$.

Assume that $\psi$ is a positive functional of the module $\mathscr{A}$ such that $g \cdot \psi=f$. If $P \psi \neq \phi$, then there is an element $A$ in $\mathscr{A}^{+}$such that $P \psi(A) \neq \phi(A)$. There is a nonzero projection $Q$ in $\mathscr{Z} P$ and an $\varepsilon>0$ such that either

$$
Q \psi(A)+\varepsilon Q \leqq \phi(A) \text { or } Q \phi(A)+\varepsilon Q \leqq Q \psi(A) \text {. }
$$

However, we have that $g(Q \phi(A))=g(Q \psi(A))$ and so $g(\varepsilon Q)=0$ in either case. This means $Q=0$. This is a contradiction. Therefore $P \psi=\phi$. Q.E.D.

A positive functional $f$ of a $C^{*}$-algebra $\mathscr{A}$ with center $\mathscr{Z}$ is said to be centrally reducible if for every positive functional $g$ of $\mathscr{A}$ majorized by $f$ there is an element $C$ in $\mathscr{Z}^{+}$such that $f(C A)=g(A)$ for every $A$ in $\mathscr{A}$. These centrally reducible functionals have been the object of much study ([5], [8], [25], [26]). The next theorem concerns these functionals. 
THEOREM 3.2. Let $\mathscr{A}$ be a von Neumann algebra with center $\mathscr{Z}$. Let $f$ be a positive functional on $\mathscr{A}$ whose restriction $g$ to the center $\mathscr{Z}$ is $\sigma$-weakly continuous. The functional $f$ is centrally reducible if and only if the unique positive functional $\phi$ of the module $\mathscr{A}$ with $g \cdot \phi=f$ and with $\phi(1)$ equal to the support $P$ of $g$ is $\mathscr{Z}$-irreducible.

Proof. Suppose $f$ is centrally reducible. Let $\psi$ be a positive functional of the module $\mathscr{A}$ which is majorized by $\phi$. Then $g \cdot \psi$ is majorized by $g \cdot \phi$. There is a $C$ in $\mathscr{Z}^{+}$with $g(C \phi(A))=g(\psi(A))$ for every $A$ in $\mathscr{A}$. By the same argument as contained in Theorem 3.1, we find that $C \phi(A)=P \psi(A)$ for every $A$ in $\mathscr{A}$. Because $0 \leqq \psi(1-P)$ $\leqq \phi(1-P)=0$ we have that $P \psi=\psi$. Therefore $C \phi=\psi$. This proves $\phi$ is $\mathscr{Z}$-irreducible.

Conversely, let $\phi$ be $\mathscr{Z}$-irreducible. Let $h$ be a positive functional on $\mathscr{A}$ majorized by $f$. The restriction of $h$ to the center of $\mathscr{A}$ is majorized by $g$. Therefore, $h$ is weakly continuous on $\mathscr{Z}$. By the Radon-Nikodym theorem there is a positive element $B$ in $\mathscr{Z} P$ such that $g(B A)=h(A)$ for every $A$ in $\mathscr{Z}$. There is by Theorem 3.1 a positive functional of the module $\mathscr{A}$ such that $h \cdot \psi=h$. Thus $g(B \psi(A))=h(A)$ for every $A$ in $\mathscr{A}$. Hence, for every $A$ in $\mathscr{A}^{+}$we find that $\phi(A)-B \psi(A) \geqq 0$. This means that $\phi$ majorizes $B \psi$. There is a $C$ in $\mathscr{Z}^{+}$such that $C \phi=B \psi$. Thus we find that $f(C A)=h(A)$ for every $A$ in $\mathscr{A}$. This proves $f$ is centrally reducible. Q.E.D.

Now let $f$ be a positive functional of the von Neumann algebra with center $\mathscr{Z}$. Suppose the restriction $g$ of $f$ to $\mathscr{Z}$ is weakly continuous. Let $\nu$ be the so-called spectral measure on the spectrum $Z$ of $\mathscr{Z}$ such that $g(A)=\int A^{\wedge}(\zeta) d \nu(\zeta)$ for every $A \in \mathscr{Z}$. Here $A^{\wedge}$ denotes the Gelfand transform of $A$. Let $\phi$ denote the unique positive functional of the module $\mathscr{A}$ such that $\phi(1)$ is the support $P$ of $g$ and such that $f=g \cdot \phi$. Then $f(A)=\int \phi(A)^{\wedge}(\zeta) d \nu(\zeta)$. We note that

(1) $\left\{\zeta \in Z \mid P^{\wedge}(\zeta)=1\right\}$ is the support of the spectral measure $\nu$;

(2) $f_{\zeta}(A)=\phi(A)^{\wedge}(\zeta)$ is a positive functional of $\mathscr{A}$ whose kernel contains [ $\zeta$;

(3) for each fixed $A$ in $\mathscr{A}$, the function $\zeta \rightarrow f_{\zeta}(A)$ is continuous on $\mathscr{Z}$.

In $\$ 4$ we shall show that

(4) $f_{\zeta}$ is irreducible if $\phi$ is $\mathscr{Z}$-irreducible.

If $\nu$ is a spectra measure and $\left\{f_{\zeta} \mid \zeta \in Z\right\}$ is a family of functions satisfying properties (1)-(3) (respectively, (1)-(4)) then the relation $f(A)=\int f_{\zeta}(A) d \nu(\zeta)$ defines a positive functional (respectively, a centrally reducible functional) which is weakly continuous on $\mathscr{Z}$ [26].

4. Representations on $A W^{*}$-modules. In this section we study the representations induced by positive module homomorphisms. Our main result will be an analogue of Kadison's Theorem [13] on strictly irreducible representations.

Let $\mathscr{A}$ be a von Neumann algebra. A positive functional $\phi$ of the module $\mathscr{A}$ will be called a state (or expectation) of the module $\mathscr{A}$ if $\phi(1)=1$. Then if $\psi$ is a positive functional of the module $\mathscr{A}$, there is a state $\phi$ of the module $\mathscr{A}$ such that $\psi=\psi(1) \phi$ [19], [24]. A state $\phi$ of the module $\mathscr{A}$ is said to be a pure state if it is an extreme point of the set of positive functionals of norm not exceeding 1 of the module $\mathscr{A}$. 
Proposition 4.1. Let $\mathscr{A}$ be a von Neumann algebra. Let $E$ be a projection in $\mathscr{A}$ and let $P$ be the central support of $E$. There is a pure state of the module $\mathscr{A}$ such that $\phi(E)=P$.

Proof. Let $\mathscr{Z}$ be the center of $\mathscr{A}$. The set $\mathscr{K}$ of states $\phi$ of the module $\mathscr{A}$ such that $\phi(E)=P$ is a $\mathscr{Z}$-convex weak*-compact subset of the set $\mathscr{S}$ of positive functionals of norm not exceeding 1 of the module $\mathscr{A}$. The set $\mathscr{K}$ is nonvoid. Indeed, let $F_{1}$ be an abelian projection in the commutator $\mathscr{Z}^{\prime}$ of $\mathscr{Z}$ with central support $P$ which is majorized by $E$. Let $F_{2}$ be an abelian projection in $\mathscr{Z}^{\prime}$ of central support $1-P$. Then $F=F_{1}+F_{2}$ is an abelian projection of central support 1. This means that $\tau_{F}$ restricted to $\mathscr{A}$ is a state. Also $\tau_{F}(E)=P$, i.e. $\tau_{F}$ is an element of $\mathscr{K}$.

Let $\phi$ be an extreme point of $\mathscr{K}$ (Theorem 2.2). We show $\phi$ is an extreme point of $\mathscr{S}$. Let $\phi_{1}$ and $\phi_{2}$ be two functionals in $\mathscr{S}$ and let $C$ be a central element strictly between 0 and 1 such that

$$
C \phi_{1}+(1-C) \phi_{2}=\phi .
$$

We have that $\phi_{j}(1) \leqq 1$ and thus, $\phi_{j}(E) \leqq \phi_{j}(P) \leqq P(j=1,2)$. Therefore,

$$
C \phi_{1}(1)+(1-C) \phi_{2}(1)=1 \text { and } C \phi_{1}(E)+(1-C) \phi_{2}(E)=P
$$

imply that $\phi_{1}(1)=\phi_{2}(1)=1$ and $\phi_{1}(E)=\phi_{2}(E)=P$. Thus, both $\phi_{1}$ and $\phi_{2}$ are elements of $\mathscr{K}$. Because $\phi$ is an extreme point of $\mathscr{K}$, we have that $\phi_{1}=\phi_{2}=\phi$. Q.E.D.

Let $\mathscr{A}$ be a von Neumann algebra and let $\phi$ be a state of $\mathscr{A}$. Let

$$
L_{\phi}=\left\{A \in \mathscr{A} \mid \phi\left(A^{*} A\right)=0\right\} \text {. }
$$

The factor set $\mathscr{A}-L_{\phi}$ is a module over $\mathscr{Z}$ which is supplied with an inner product $\left(A-L_{\phi}, B-L_{\phi}\right)=\phi\left(B^{*} A\right)$ with values in $\mathscr{Z}$. The space $\mathscr{A}-L_{\phi}$ can then be embedded in a faithful $A W^{*}$-module $M_{\phi}$ over $\mathscr{Z}$ obtained by completing $\mathscr{A}-L_{\phi}$ in the following way. The set $M_{\phi}$ is the norm completion of the set of all $\left\{A_{n}-L_{\phi}, P_{n}\right\}_{n}$, where $\left\{P_{n}\right\}$ is a set of orthogonal central projections of sum 1 and $\left\{A_{n}-L_{\phi}\right\}$ is a set of elements of $\mathscr{A}-L_{\phi}$ with $\left\{\phi\left(A_{n}^{*} A_{n}\right)\right\}$ bounded in $\mathscr{Z}$, supplied with the norm induced by the inner product

$$
\left\langle\left\{A_{n}-L_{\phi}, P_{n}\right\},\left\{B_{m}-L_{\phi}, Q_{m}\right\}\right\rangle=\sum_{m, n} \phi\left(B_{m}^{*} A_{n}\right) P_{n} Q_{m} .
$$

There is a bounded homomorphism $\pi_{\phi}$ of $\mathscr{A}$, which is also a module homomorphism over $\mathscr{Z}$, into the algebra $L\left(M_{\phi}\right)$ of all bounded module homomorphisms of $M_{\phi}$ onto itself that extends the left multiplication representation of $\mathscr{A}$ on $\mathscr{A}-L_{\phi}$. This map $\pi_{\phi}$ is called the canonical representation of $\mathscr{A}$ on $M_{\phi}$ induced by $\phi$. For the operators $T$ in $L\left(M_{\phi}\right)$ an involution $T \rightarrow T^{*}$ of $L\left(M_{\phi}\right)$ is defined. We have the relation $\langle T A, B\rangle=\left\langle A, T^{*} B\right\rangle$ for $A$ and $B$ in $M_{\phi}$. The involution also satisfies the relation $\left\|T^{*} T\right\|=\|T\|^{2}$. Finally, the representation $\pi_{\phi}$ preserves adjoints in the sense that $\pi_{\phi}\left(A^{*}\right)=\pi_{\phi}(A)^{*}$ for every $A$ in $\mathscr{A}$ ([17], [6], [28]).

If $\mathscr{Z}$ is a commutative von Neumann algebra on a Hilbert space $H$ and if $\mathscr{Z}^{\prime}$ is the commutator of $\mathscr{Z}$ on $H$, then for any abelian projection $E$ of $\mathscr{Z}^{\prime}$ of central 
support 1 the module $\mathscr{Z}^{\prime} E$ is an $A W^{*}$-module over $\mathscr{Z}$. The inner product is defined to be $\langle A, B\rangle=\tau_{E}\left(B^{*} A\right)$ for $A$ and $B$ in $\mathscr{Z}^{\prime} E$ [17].

A specific form for $M_{\phi}$ is now obtained.

Proposition 4.2. Let $\mathscr{A}$ be a von Neumann algebra with center $\mathscr{Z}$ and let $\phi$ be a state of the module $\mathscr{A}$. There is a Hilbert space $H$ and a representation $\pi$ of $\mathscr{A}$ on $H$ with the following properties:

(1) $\pi$ is faithful on $\mathscr{Z}$;

(2) $\pi(\mathscr{Z})$ is a von Neumann algebra on $H$;

(3) there is an abelian projection $E$ in the commutator $\pi(\mathscr{Z})^{\prime}$ of $\pi(\mathscr{Z})$ on $H$ such that $\pi(\phi(A))=\tau_{E}(\pi(A))$; and

(4) there is a function $\Phi$ of $M_{\phi}$ onto the completion of the module $\pi(\mathscr{A}) E$ in $\pi(\mathscr{Z})^{\prime} E$ such that

$$
\Phi\left(A_{1} B_{1}+A_{2} B_{2}\right)=\pi\left(A_{1}\right) \Phi\left(B_{1}\right)+\pi\left(A_{2}\right) \Phi\left(B_{2}\right)
$$

for every $A_{1}$ and $A_{2}$ in $\mathscr{Z}$ and every $B_{1}$ and $B_{2}$ in $M_{\phi}$;

$$
\pi(\langle A, B\rangle)=\langle\Phi(A), \Phi(B)\rangle
$$

for every $A$ and $B$ in $M_{\phi}$; and $\Phi\left(\pi_{\phi}(A) B\right)=\pi(A) \Phi(B)$ for every $A$ in $\mathscr{A}$ and $B$ in $M_{\phi}$. If $\phi$ is a pure state of the module $\mathscr{A}$, then

(5) the commutator of $\pi(\mathscr{A})$ on $H$ is equal to $\pi(\mathscr{Z})$.

Proof. Let $\left\{P_{n}\right\}$ be a set of nonzero mutually orthogonal projections of $\mathscr{A}$ with sum equal to 1 such that each algebra $\mathscr{Z} P_{n}$ is $\sigma$-finite. Let $x_{n}$ be a unit vector of the Hilbert space of $\mathscr{Z} P_{n}$ which separates $\mathscr{Z} P_{n}\left[1, I, \S 2\right.$, No. 1]. Let $\pi_{n}$ be the canonical representation of $\mathscr{A}$ on the Hilbert space $H_{n}$ induced by the positive functional $w_{x_{n}} \cdot \phi$ of $\mathscr{A}$. Here $w_{x}(A)=(A x, x)$ for any vector $x$. Let $y_{n}$ be a vector in $H_{n}$ cyclic under $\pi_{n}(A)$ such that

$$
\left(\pi_{n}(A) y_{n}, y_{n}\right)=w_{x_{n}}(\phi(A)) .
$$

Let $\pi$ be the representation $\pi=\sum \oplus \pi_{n}$ on the Hilbert space $H=\sum \oplus H_{n}$.

We show that $\pi$ is faithful on $\mathscr{Z}$. Indeed, if $A \in \mathscr{Z}$ and $\pi(A)=0$, then $\pi\left(A P_{n}\right)=0$ for every $n$. This means $\pi_{n}\left(A P_{n}\right)=0$. However, the representation $\pi_{n}$ is faithful on $\mathscr{Z} P_{n}$; hence $A P_{n}=0$ for every $n$. This means $A=0$. Thus $\pi$ is faithful on $\mathscr{Z}$.

We prove now that $\pi$ is $\sigma$-weakly continuous when restricted to $\mathscr{Z}$. Let $\left\{A_{m}\right\}$ be a monotonely increasing net $\mathscr{Z}^{+}$with least upper bound $A$. We have (Proposition $3.1)$ that $\left\{\pi_{n}\left(A_{m}\right)\right\}_{m}$ converges strongly to $\pi_{n}(A)$ for each $n$. Now let $x$ be an element in $H$ and let $\varepsilon>0$ be given. There is a finite subset $P_{1}, P_{2}, \ldots, P_{k}$ of $\left\{P_{n}\right\}$ of $\operatorname{sum} P$ such that $\|x-\pi(P) x\| \leqq \varepsilon$ because each $\pi\left(P_{n}\right)$ is the projection of $H$ on $H_{n}$. Suppose that for $m \geqq m_{0}$ we have that

$$
\left\|\left(\pi_{j}\left(A_{m}\right)-\pi_{j}(A)\right) \pi\left(P_{j}\right) x\right\| \leqq \varepsilon k^{-1} \quad \text { for } j=1, \ldots, k .
$$


Then

$$
\begin{aligned}
\left\|\pi(A) x-\pi\left(A_{m}\right) x\right\| & \leqq\left\|\left(\pi(A)-\pi\left(A_{m}\right)\right)(1-\pi(P)) x\right\|+\left\|\left(\pi(A)-\pi\left(A_{m}\right)\right) \pi(P) x\right\| \\
& \leqq 2\|A\| \varepsilon+\sum\left\{\left\|\left(\pi_{j}(A)-\pi_{j}\left(A_{m}\right)\right) \pi_{j}\left(P_{j}\right) x\right\| \mid 1 \leqq j \leqq k\right\} \\
& \leqq(2\|A\|+1) \varepsilon .
\end{aligned}
$$

This proves that $\pi$ is a $\sigma$-weakly continuous isomorphism of $\mathscr{Z}$.

By the proof of Theorem 3.1 there is for each $n$ an abelian projection $E_{n}^{\prime}$ in the commutator $\pi_{n}\left(\mathscr{Z} P_{n}\right)^{\prime}$ on $H_{n}$ associated with the subspace

$$
\text { closure }\left\{\pi(A) y_{n} \mid A \in \mathscr{Z}\right\}
$$

such that

$$
\tau_{E_{n}^{\prime}}^{\prime}\left(\pi_{n}(A)\right)=\pi_{n}\left(\phi\left(A P_{n}\right)\right)
$$

Since $\pi(\mathscr{Z})^{\prime} \pi\left(P_{n}\right)$ is the commutator of $\pi(\mathscr{Z}) \pi\left(P_{n}\right)$ on $H_{n}$, we have that there is an abelian projection $E_{n}$ in the von Neumann algebra $\pi(\mathscr{Z})^{\prime}$ on $H$ majorized by $\pi\left(P_{n}\right)$ such that

$$
\tau_{E_{n}}\left(\pi\left(A P_{n}\right)\right)=\pi\left(\phi\left(A P_{n}\right)\right)
$$

Let $E$ be the abelian projection in $\pi(\mathscr{Z})^{\prime}$ given by $E=\sum E_{n}$. Then

$$
\begin{aligned}
\tau_{E}(\pi(A)) \pi\left(P_{n}\right) & =\tau_{E_{n}}\left(\pi\left(A P_{n}\right)\right)=\pi\left(\phi\left(A P_{n}\right)\right) \\
& =\pi(\phi(A)) \pi\left(P_{n}\right) \quad \text { for every } n .
\end{aligned}
$$

This proves that $\tau_{E}(\pi(A))=\pi(\phi(A))$ for every $A$ in $\mathscr{A}$.

Let $\left\{A_{n}-L_{\phi} \mid n \in N\right\}$ and $\left\{B_{m}-L_{\phi} \mid m \in N^{\prime}\right\}$ be two bounded sets in $\mathscr{A}-L_{\phi}$ and let $\left\{Q_{n} \mid n \in N\right\}$ and $\left\{R_{m} \mid m \in N^{\prime}\right\}$ be two sets of mutually orthogonal central projections of sum 1 respectively. Then $\sum \pi\left(Q_{n}\right) \pi\left(A_{n}\right) E$ and $\sum \pi\left(R_{m}\right) \pi\left(B_{m}\right) E$ are elements of the $A W^{*}$-module $\pi(\mathscr{Z})^{\prime} E$. We have that

$$
\begin{aligned}
\pi\left(\left\langle\sum Q_{n}\left(A_{n}-L_{\phi}\right), \sum R_{m}\left(B_{m}-L_{\phi}\right)\right\rangle\right) & =\pi\left(\sum_{m, n} Q_{n} R_{m} \phi\left(B_{m}^{*} A_{n}\right)\right) \\
& =\left\langle\sum \pi\left(Q_{n}\right) \pi\left(A_{n}\right) E, \sum \pi\left(R_{m}\right) \pi\left(B_{m}\right) E\right\rangle
\end{aligned}
$$

in the respective inner products of $M_{\phi}$ and $\pi(\mathscr{Z})^{\prime} E$. Therefore,

$$
\Phi\left(\sum Q_{n}\left(A_{n}-L_{\phi}\right)\right)=\sum \pi\left(Q_{n}\right) \pi\left(A_{n}\right) E
$$

defines a function of a uniformly dense submodule

$$
\begin{aligned}
M_{1}=\left\{\sum Q_{n}\left(A_{n}-L_{\phi}\right) \mid\right. & \left\{Q_{n}\right\} \text { is a set of mutually orthogonal } \\
& \text { central projections of sum } 1 ; \\
& \left.\left\{A_{n}-L_{\phi}\right\} \text { is a bounded set in } \mathscr{A}-L_{\phi}\right\}
\end{aligned}
$$

of the module $M_{\phi}$ into the submodule 


$$
\begin{aligned}
M_{2}=\left\{\sum \pi\left(Q_{n}\right) \pi\left(A_{n}\right) E \mid\right. & \left\{\pi\left(Q_{n}\right)\right\} \text { is a set of mutually orthogonal } \\
& \text { projections of } \pi(\mathscr{Z}) ; \\
& \left.\left\{\pi\left(A_{n}\right) E\right\} \text { is a bounded subset of } \pi(\mathscr{A}) E\right\}
\end{aligned}
$$

of the module $\pi(\mathscr{Z})^{\prime} E$.

We have that $\Phi$ is a linear function of $M_{1}$ into $M_{2}$ such that $\Phi(A B)=\pi(A) \Phi(B)$ for every $A$ in $\mathscr{Z}$ and $B$ in $M_{1}$. The range of $\Phi$ is $M_{2}$. There is a unique extension of $\Phi$ to a map which we again call $\Phi$ of the norm completion $M_{\phi}$ of $M_{1}$ onto the closure of $M_{2}$ in $\pi(\mathscr{Z})^{\prime} E$ such that

$$
\Phi\left(A_{1} B_{1}+A_{2} B_{2}\right)=\pi\left(A_{1}\right) \Phi\left(B_{1}\right)+\pi\left(A_{2}\right) \Phi\left(B_{2}\right)
$$

for every $A_{1}$ and $A_{2}$ in $\mathscr{Z}$ and $B_{1}$ and $B_{2}$ in $M_{\phi}$ and such that

$$
\langle\Phi(A), \Phi(B)\rangle=\pi(\langle A, B\rangle)
$$

for every $A$ and $B$ in $M_{\phi}$. Since the closure of $M_{2}$ is precisely the $A W^{*}$-module generated by $\pi(\mathscr{A}) E$ in $\pi(\mathscr{Z})^{\prime} E$ [6, Lemma 4.1], we have that the range of $\Phi$ is the $A W^{*}$-module generated by $\pi(\mathscr{A}) E$.

Finally, let $\left\{A_{n}-L_{\phi}\right\}$ be a bounded set in $\mathscr{A}-L_{\phi}$ and let $\left\{Q_{n}\right\}$ be a set of mutually orthogonal central projections of sum 1 . Then

$$
\begin{aligned}
\Phi\left(\pi_{\phi}(A)\left(\sum Q_{n}\left(A_{n}-L_{\phi}\right)\right)\right) & =\Phi\left(\sum Q_{n}\left(A A_{n}-L_{\phi}\right)\right) \\
& =\sum \pi\left(Q_{n}\right) \pi\left(A A_{n}\right) E=\pi(A) \sum \pi\left(Q_{n}\right) \pi\left(A_{n}\right) E \\
& =\pi(A) \Phi\left(\sum Q_{n}\left(A_{n}-L_{\phi}\right)\right)
\end{aligned}
$$

for every $A$ in $\mathscr{A}$. Thus we have that $\Phi\left(\pi_{\phi}(A) B\right)=\pi(A) \Phi(B)$ for every $A$ in $\mathscr{A}$ and $B$ in $M_{\phi}$. This completes the proof of (4).

Now assume $\phi$ is a pure state. Let $\eta$ be the inverse of $\pi$ restricted to $\mathscr{Z}$. Let $A$ be a positive element in the unit sphere of the commutator, $\pi(\mathscr{A})^{\prime}$ of $\pi(\mathscr{A})$ on $H$. Let $\tau=\tau_{E}$. The relation

$$
\eta(\tau(A \pi(B)))=\psi(B)
$$

defines a functional of the module $\mathscr{A}$. For every $B$ in $\mathscr{A}$ we have that

$$
\psi\left(B^{*} B\right)=\eta\left(\tau\left(A^{1 / 2} \pi\left(B^{*} B\right) A^{1 / 2}\right)\right) \geqq 0
$$

and

$$
\begin{aligned}
\psi\left(B^{*} B\right) & =\eta\left(\tau\left(\pi\left(B^{*} B\right)^{1 / 2} A \pi\left(B^{*} B\right)^{1 / 2}\right)\right) \\
& \leqq \eta\left(\tau\left(\pi\left(B^{*} B\right)\right)\right)\|A\| \leqq \phi\left(B^{*} B\right)
\end{aligned}
$$

So $\psi$ is a positive functional majorized by $\phi$. There is a $C$ in $\mathscr{Z}^{+}$such that $C \phi=\psi$ (cf. §2). So for every $B_{1}$ and $B_{2}$ in $\mathscr{A}$ we have that

$$
\tau\left(\pi\left(B_{2}\right)^{*}(A-\pi(C)) \pi\left(B_{1}\right)\right)=0 .
$$


This means that

$$
\left((A-\pi(C)) \pi\left(B_{1}\right) y_{n}, \pi\left(B_{2}\right) y_{m}\right)=0
$$

for every $y_{n}$ and $y_{m}$. However, the closure of the linear span of

$$
\left\{\pi(B) y_{n} \mid B \text { in } \mathscr{A}, \text { all } y_{n}\right\}
$$

is $H$. Thus $A=\pi(C)$. Therefore $\pi(\mathscr{A})^{\prime}$ is equal to $\pi(\mathscr{Z})$. Q.E.D.

Before continuing we present a brief discussion of a certain trace that is particularly useful. Let $\mathscr{A}$ be a type I algebra with center $\mathscr{Z}$. There is a locally compact space $X$ and a positive measure $\nu$ on $X$ of support $X$ such that $\mathscr{Z}$ is isometric *-isomorphic to the algebra $L^{\infty}(X, \nu)$ of all essentially bounded complex-valued measurable functions on $X$. Identify $\mathscr{Z}$ with $L^{\infty}(X, v)$. There is a function $\operatorname{Tr}$ of $\mathscr{A}^{+}$ into the set of all positive finite or infinite valued measurable functions on $X$ with the following properties:

(1) $\operatorname{Tr}\left(C_{1} A_{1}+C_{2} A_{2}\right)=C_{1} \operatorname{Tr}\left(A_{1}\right)+C_{2} \operatorname{Tr}\left(A_{2}\right)$ for $C_{1}, C_{2}$ in $\mathscr{Z}^{+}$and $A_{1}, A_{2}$ in $\mathscr{A}^{+}$

(2) $\operatorname{Tr}\left(U^{*} A U\right)=\operatorname{Tr}(A)$ for every $A$ in $\mathscr{A}^{+}$and every unitary $U$ in $\mathscr{A}$;

(3) if $\left\{A_{n}\right\}$ is a monotonely increasing net in $\mathscr{A}^{+}$with least upper bound $A$, then $\left\{\operatorname{Tr}\left(A_{n}\right)\right\}$ has least upper bound $\operatorname{Tr}(A)$;

(4) $\operatorname{Tr}(E)=\tau_{E}(E)$ for every abelian projection $E$ in $\mathscr{A}$.

If $\mathscr{P}=\left\{A \in \mathscr{A}^{+} \mid \operatorname{Tr}(A) \in \mathscr{Z}^{+}\right\}$, then $\mathscr{P}$ is the set of all positive elements of a two-sided ideal $\mathscr{T}$ in $\mathscr{A}$ called the trace class of $\mathscr{A}$. In particular every abelian projection is a member of $\mathscr{T}$. The function $\operatorname{Tr}$ on $\mathscr{P}=\mathscr{T} \cap \mathscr{A}^{+}$may be extended to a linear function $\operatorname{Tr}$ of $\mathscr{T}$ into $\mathscr{Z}$ which is also a module homomorphism. For every $A \in \mathscr{T}$ the function $B \rightarrow \operatorname{Tr}(A B)$ is a function of $\mathscr{A}^{\sim}$ which is also continuous in the respective $\sigma$-weak topologies. We have that $\operatorname{Tr}(B A)=\operatorname{Tr}(A B)$ for every $A$ in $\mathscr{T}$ and $B$ in $\mathscr{A}$. Also we have that $\|B\|^{2} \leqq\left\|\operatorname{Tr}\left(B^{*} B\right)\right\|$ for every $B$ in $\mathscr{T}[9, \S 4]$.

Let $M$ be an $A W^{*}$-module over the commutative $A W^{*}$-algebra $\mathscr{Z}$ and let $\mathscr{B}$ be a subalgebra of the algebra $L(M)$ of all bounded linear operators on $M$. The algebra $\mathscr{B}$ is said to be irreducible on $M$ if given $A$ in $L(M)$ and $C_{1}, C_{2}, \ldots, C_{n}$ in $M$ then there is a $B$ in $\mathscr{B}$ such that $B C_{j}=A C_{j}$ for every $j=1,2, \ldots, n$.

THEOREM 4.3. Let $\mathscr{A}$ be a von Neumann algebra with center $\mathscr{Z}$. Let $\phi$ be a pure state of the module $\mathscr{A}$. Then the module $M_{\phi}$ induced by $\phi$ is equal to $\mathscr{A}-L_{\phi}$ and $\pi_{\phi}(\mathscr{A})$ is irreducible on $M_{\phi}$.

Proof. Let $\pi$ be the representation relative to $\phi$ of $\mathscr{A}$ on the Hilbert space $H$ constructed in Proposition 4.2. Then $\pi$ enjoys properties (1)-(5) of this proposition. Let $E$ be an abelian projection of the commutator $\mathscr{B}$ of $\pi(\mathscr{Z})$ on $H$ such that $\tau_{E}(\pi(A))=\pi(\phi(A))$. We show that $\pi(\mathscr{A}) E=\mathscr{B} E$. This means that the module $M_{\phi}$ is $\mathscr{A}-L_{\phi}$. The algebra of all bounded linear operators on $\mathscr{B} E$ is identified with $\mathscr{B}$ acting on $\mathscr{B} E$ by left multiplication [17, Theorem 8]. Given $B_{1}, B_{2}, \ldots, B_{m}$ and $B$ in $\mathscr{B}$ we show that there is an $A$ in $\mathscr{A}$ with $\pi(A) B_{j} E=B B_{j} E$ for $j=1,2, \ldots, m$. We 
may also show that $A$ can be chosen to be self-adjoint if $B$ is self-adjoint. The proof essentially consists of showing that $E$ is a regular projection with respect to $\pi(\mathscr{A})[27]$ using a construction known for pure states (cf. [2, §2.8]).

As a preliminary step assume that $B_{1} E, B_{2} E, \ldots, B_{m} E$ are partial isometric operators $V_{1}, V_{2}, \ldots, V_{m}$ respectively. Assume also that the range projections $F_{1}, F_{2}, \ldots, F_{m}$ of the $V_{1}, V_{2}, \ldots, V_{m}$ are mutually orthogonal. We show that there is an element $B^{\prime}$ in $\mathscr{B}$ such that $B^{\prime} V_{i}=B V_{i}(1 \leqq i \leqq m)$ and such that $\left\|B^{\prime}\right\|^{2}$ $\leqq 2 \sum\left\|V_{i}^{*} B^{*} B V_{i}\right\|$. We show that $B^{\prime}$ may be chosen to be self-adjoint if $B$ is selfadjoint. Let $G_{i}$ be the range projection of $B V_{i}(1 \leqq i \leqq m)$. Since $G_{i}$ is equivalent to the domain projection of $B V_{i}$, which is majorized by $E$, the projection $G_{i}$ is abelian (cf. [1, III, §1]). Let $G$ be the least upper bound of the set

$$
\left\{F_{i} \mid 1 \leqq i \leqq m\right\} \cup\left\{G_{i} \mid 1 \leqq i \leqq m\right\} .
$$

The projection $G-\sum F_{i}$ may be written as the sum of mutually orthogonal abelian projections $F_{m+1}, F_{m+2}, \ldots, F_{p}$ (cf. [9, Theorem 2.1]). Let

$$
B^{\prime}=\sum\left\{F_{j} B F_{i} \mid 1 \leqq i \leqq m ; 1 \leqq j \leqq p\right\}
$$

if $B$ is not self-adjoint and let

$$
B^{\prime}=\sum\left\{F_{j} B F_{i} \mid 1 \leqq i \leqq p ; 1 \leqq j \leqq m\right\}+\sum\left\{F_{j} B F_{i} \mid 1 \leqq i \leqq m ; m+1 \leqq j \leqq p\right\}
$$

if $B$ is self-adjoint. In this case $B^{\prime}$ is self-adjoint. In either case

$$
B^{\prime} V_{i}=\sum\left\{F_{j} B V_{i} \mid 1 \leqq j \leqq p\right\}=B V_{i}
$$

for $i=1,2, \ldots, m$. In the first case

$$
\begin{aligned}
\operatorname{Tr}\left(B^{*} B^{\prime}\right) & =\sum\left\{\operatorname{Tr}\left(F_{i} B^{*} B^{\prime} F_{i}\right) \mid 1 \leqq i \leqq m\right\} \\
& =\sum\left\{\tau_{F_{i}}\left(B^{\prime *} B^{\prime}\right) \mid 1 \leqq i \leqq m\right\}
\end{aligned}
$$

In the second case we have that

$$
\begin{aligned}
\operatorname{Tr}\left(B^{\prime *} B^{\prime}\right)= & \operatorname{Tr}\left(B^{\prime 2}\right)=\sum\left\{\operatorname{Tr}\left(F_{i}{B^{\prime}}^{2} F_{i}\right) \mid 1 \leqq i \leqq m\right\} \\
& +\sum\left\{\operatorname{Tr}\left(F_{j} B^{\prime} F_{i} B^{\prime} F_{j}\right) \mid m+1 \leqq i \leqq p ; 1 \leqq j \leqq p\right\} \\
= & \sum\left\{\operatorname{Tr}\left(F_{i}{B^{\prime 2}}^{2} F_{i}\right) \mid 1 \leqq i \leqq m\right\} \\
& +\sum\left\{\operatorname{Tr}\left(F_{j} B^{\prime} F_{i} B^{\prime} F_{j}\right) \mid m+1 \leqq i \leqq p ; 1 \leqq j \leqq m\right\} \\
\leqq & 2 \sum\left\{\tau_{F_{i}}\left(B^{\prime 2}\right) \mid 1 \leqq i \leqq m\right\}
\end{aligned}
$$

since $F_{j} B^{\prime}\left(\sum\left\{F_{i} \mid m+1 \leqq i \leqq p\right\}\right) B^{\prime} F_{j} \leqq F_{j}{B^{\prime}}^{2} F_{j}$.

We have that

$$
\left\|\tau_{F_{i}}\left(B^{\prime *} B^{\prime}\right)\right\|=\left\|F_{i} B^{*} B^{\prime} F_{\imath}\right\|=\left\|V_{i}^{*} B^{*} B^{\prime} V_{i}\right\| .
$$

Thus in either case we conclude that

$$
\left\|B^{\prime *} B^{\prime}\right\| \leqq\left\|\operatorname{Tr}\left(B^{* *} B^{\prime}\right)\right\| \leqq 2 \sum\left\|V_{i}^{*} B^{*} B V_{i}\right\| .
$$


This verifies the existence of $B^{\prime}$ in $\mathscr{B}$. So we may assume that

$$
\|B\| \leqq(2 m)^{1 / 2} \alpha \quad \text { where } \alpha=\max \left\{\left\|B V_{i}\right\| \mid 1 \leqq i \leqq m\right\} .
$$

By an application of Tomita's results [27, Theorem 6] we may find a nonzero projection $F$ in $\mathscr{B}$ majorized by $E$ and an element $A$ in $\pi(\mathscr{A})$ such that $\|A\| \leqq$ $2(2 m)^{1 / 2} \alpha$ and $A V_{j} F=B V_{j} F$ for $j=1,2, \ldots, m$. Indeed given a unit vector $x$ in the Hilbert space of $\mathscr{B}$ such that $E x=x$, then we may construct by induction a decreasing sequence $\left\{F_{n}^{\prime}\right\}$ of abelian projections and a sequence of elements $\left\{A_{n}\right\}$ in $\pi(\mathscr{A})$ such that

(1) $\left\|F_{n}^{\prime} x-F_{n+1}^{\prime} x\right\| \leqq 4^{-n+1}$ and $\left\|x-F_{1}^{\prime} x\right\| \leqq 4^{-1}$;

(2) $\left\|A_{n}\right\| \leqq 2^{-n+1}(2 m)^{1 / 2} \alpha$; and

(3) lub $\left\{\left\|\left(\sum\left\{A_{j}: 1 \leqq j \leqq n\right\}-B\right) V_{i} F_{n}^{\prime}\right\|: 1 \leqq i \leqq m\right\} \leqq 2^{-n} \alpha$ for every $n=1,2, \ldots$

Then $A=\sum A_{n}$ and $F=$ glb $F_{n}^{\prime} \neq 0$. If $B$ is self-adjoint then $A$ may be chosen selfadjoint. Let $\left\{P_{n} \mid n \in D\right\}$ be a maximal set of mutually orthogonal nonzero projections in $\pi(\mathscr{Z})$ with the property: for each $P_{n}$ there is an element $A_{n}$ in $\pi(\mathscr{A}) P_{n}$ such that $\left\|A_{n}\right\| \leqq 2(2 m)^{1 / 2} \alpha$ and such that $A_{n} V_{j} E=B V_{j} E P_{n}$. We see that $\sum P_{n}=1$; otherwise, the projection $P=1-\sum P_{n}$ is nonzero. There is a nonzero projection $F$ majorized by $E P$ and an element $A$ in $\pi(\mathscr{A})$ such that $\|A\| \leqq 2(2 m)^{1 / 2} \alpha$ and $A V_{j} F$ $=B V_{j} F$. But there is a nonzero projection $Q$ in $\pi(\mathscr{Z})$ majorized by $P$ such that $Q E=F$. This contradicts the maximality of the set $\left\{P_{n}\right\}$. Therefore, the least upper bound of the set $\left\{P_{n}\right\}$ is 1 . There is a set $\left\{Q_{n} \mid n \in D\right\}$ of mutually orthogonal projections in $\mathscr{Z}$ such that $\pi\left(Q_{n}\right)=P_{n}$ for each $n \in D$. Since $\pi$ is norm decreasing, there is for each $A_{n}$ an element $B_{n}$ in $\mathscr{A} P_{n}$ of norm not exceeding $3\left(2 m^{1 / 2}\right) \alpha$ such that $\pi\left(B_{n}\right)=A_{n}$. There is an $A$ in $\mathscr{A}$ such that $A Q_{n}=B_{n}$ for each $n$ in $D$. For each $j=1$, $2, \ldots, m$ we have $\pi(A) V_{j} E=B V_{j} E$ because $\pi(A) V_{j} E P_{n}=B V_{j} E P_{n}$ for every $n$ in $D$.

Let us now assume that $B_{1} E, B_{2} E, \ldots, B_{m} E$ are arbitrary. Let $F_{1}, F_{2}, \ldots, F_{m}$ be the range projections of $B_{1} E, B_{2} E, \ldots, B_{m} E$ respectively. Let $F$ be the least upper bound of $F_{1}, F_{2}, \ldots, F_{m}$. There are mutually orthogonal abelian projections $G_{1}, G_{2}, \ldots, G_{p}$ of sum $F$. Let $V_{1}, V_{2}, \ldots, V_{p}$ be partial isometries with range support $G_{1}, G_{2}, \ldots, G_{p}$ respectively and domain support majorized by $E$ (cf. [1, Chapter III, §3, Lemma 1]). By the first part of the proof there is an element $A$ in $\mathscr{A}$, which may be chosen to be self-adjoint if $B$ is self-adjoint, such that $\pi(A) V_{j}$ $=B V_{j}(1 \leqq j \leqq p)$. We have that $G B_{j} E=B_{j} E(1 \leqq j \leqq m)$. So

$$
\begin{aligned}
B_{j} E & =\sum\left\{G_{k} B_{j} E \mid 1 \leqq k \leqq p\right\}=\sum\left\{V_{k} V_{k}^{*} B_{j} E \mid 1 \leqq k \leqq p\right\} \\
& =\sum\left\{\tau_{E}\left(V_{k}^{*} B_{j}\right) V_{k} \mid 1 \leqq k \leqq p\right\} .
\end{aligned}
$$

Thus, we obtain

$$
B B_{j} E=\sum \tau_{E}\left(V_{k}^{*} B_{j}\right) B V_{k}=\sum \tau_{E}\left(V_{k}^{*} B_{j}\right) \pi(A) V_{k}=\pi(A) B_{j} E
$$

for $j=1,2, \ldots, m$. Q.E.D.

In the corollary we use the following ideas. Let $\mathscr{A}$ be a von Neumann algebra with center $\mathscr{Z}$; let $\zeta$ be a maximal ideal of $\mathscr{Z}$. The smallest closed two-sided ideal of 


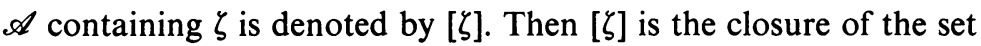

$$
\left\{\sum\left\{A_{i} B_{i} \mid 1 \leqq i \leqq n\right\} \mid A_{i} \in \zeta, B_{i} \in \mathscr{A}(1 \leqq i \leqq n) ; n=1,2, \ldots\right\} .
$$

Let $\mathscr{A}(\zeta)$ be the factor $C^{*}$-algebra $\mathscr{A} /[\zeta]$ and let $A(\zeta)$ denote the image of $A$ in $\mathscr{A}(\zeta)$. Then J. Glimm proved that for each fixed $A$ in $\mathscr{A}$ the function $\zeta \rightarrow\|A(\zeta)\|$ is continuous on the spectrum of $\mathscr{Z}[3$, Lemma 10]. If $P$ is a projection of $\mathscr{Z}$, then

$$
\|A P\|=\operatorname{lub}\left\{\|A(\zeta)\| \mid \zeta \text { in the spectrum of } \mathscr{Z} \text { and } P^{\wedge}(\zeta)=1\right\} \text {. }
$$

The least upper bound is attained. If $A(\zeta)$ is a positive element in $\mathscr{A}(\zeta)$ for each $\zeta$, then $A$ is positive in $\mathscr{A}$.

Now assume $\mathscr{A}$ is a type I algebra. Let the notation be the same as the preceding paragraph. Let $\zeta$ be a fixed maximal ideal of $\mathscr{Z}$. Suppose $E$ is an abelian projection in $\mathscr{A}$ such that $E(\zeta) \neq 0$. The space $H(\zeta)=\mathscr{A} E(\zeta)$ is a Hilbert space with the inner product $\langle A E(\zeta), B E(\zeta)\rangle=\tau_{E}\left(B^{*} A\right)^{\wedge}(\zeta)$. The algebra $\mathscr{A}$ has a representation $\Psi$ with kernel [ $\zeta$ ] on the algebra of all bounded operators on $H(\zeta)$ given by $\Psi(A) B E(\zeta)$ $=A E B(\zeta)$, for every $A$ and $B$ in $\mathscr{A}$. The closed two-sided ideal $I_{a}$ of $\mathscr{A}$ generated by the abelian projections of $\mathscr{A}$ maps onto the ideal of completely continuous operators of $H(\zeta)$. In particular if $x$ is an arbitrary vector in $H(\zeta)$ there is an abelian projection $F$ in $\mathscr{A}$ such that $\Psi(F) x=x$. The images of abelian projections under $\Psi$ have dimension not exceeding $1[3, \S 4]$.

COROllaRY. Let $\mathscr{A}$ be a von Neumann algebra with center $\mathscr{Z}$ and let $\phi$ be a $\mathscr{Z}$-irreducible functional of the module $\mathscr{A}$. For every $\zeta$ in the spectrum of $\mathscr{Z}$ the functional $\phi(A)^{\wedge}(\zeta)$ of $\mathscr{A}$ is irreducible. In particular if $\phi$ is an extreme point of the set of positive functionals of norm not exceeding 1 of the module $\mathscr{A}$, then $\phi(A)^{\wedge}(\zeta)$ is irreducible on $\mathscr{A}$.

Proof. We may assume that $\phi(1)^{\wedge}(\zeta) \neq 0$. There is a projection $P$ in $\mathscr{Z}$ which does not lie in the maximal ideal $\zeta$ of $\mathscr{Z}$ and a number $\alpha>0$ such that $\phi(1) P \geqq \alpha P$. Let $C$ be a positive element in $\mathscr{Z} P$ such that $C \phi(1)=P$. The functional $\psi=C \phi$ is a $\mathscr{Z}$ irreducible functional of the module $\mathscr{A}$. Indeed, if $\psi$ majorizes the positive functional $\theta$ of the module $\mathscr{A}$, then $P \phi$ majorizes $P \phi(1) \theta$ and so $\phi$ majorizes $P \phi(1) \theta$. There is a $D$ in $\mathscr{Z}^{+}$such that $D \phi=P \phi(1) \theta$. Thus $D \psi=C D \phi=C P \phi(1) \theta=\theta$. This proves that $\psi$ is $\mathscr{Z}$-irreducible. Since the functional $\psi(A)^{\wedge}(\zeta)$ is equal to a nonzero scalar multiple $C^{\wedge}(\zeta)$ of $\phi(A)^{\wedge}(\zeta)$, it is sufficient to prove that $\psi(A)^{\wedge}(\zeta)$ is irreducible.

Now let $\psi_{1}$ be any pure state of the module $\mathscr{A}$. The functional $P \psi+(1-P) \psi_{1}=\psi^{\prime}$ is a $\mathscr{Z}$-irreducible state of the module $\mathscr{A}$. Indeed, if $\theta$ is a positive functional of the module $\mathscr{A}$ majorized by $\psi^{\prime}$, then $P \psi=\psi$ majorizes $P \theta$ and $(1-P) \psi_{1}$ majorizes $(1-P) \theta$. There are elements $C_{1}$ and $C_{2}$ in $\mathscr{Z}^{+}$with $C_{1} \psi=P \theta$ and $C_{2} \psi_{1}=(1-P) \theta$. We may assume that $P C_{1}=C_{1}$ and $(1-P) C_{2}=C_{2}$. Setting $C=C_{1}+C_{2}$ we have that $C \psi^{\prime}=\theta$. So $\psi^{\prime}$ is a $\mathscr{Z}$-irreducible state of the module $\mathscr{A}$, i.e. $\psi^{\prime}$ is a pure state of $\mathscr{A}$. Since $\psi^{\prime}(A)^{\wedge}(\zeta)=\psi(A)^{\wedge}(\zeta)$ for every $A$ in $\mathscr{A}$ there is no loss of generality in assuming that $\psi$ is a pure state of the module $\mathscr{A}$. 
Let $\pi$ be a representation of $\mathscr{A}$ on a Hilbert space $H$ constructed in Proposition 4.2 relative to $\phi$. Let $E$ be a maximal abelian projection of the von Neumann algebra $\mathscr{B}$ generated by $\pi(\mathscr{A})$ on $H$ such that $\tau_{E}(\pi(A))=\pi(\phi(A))$ for every $A$ in $\mathscr{A}$. There is a homeomorphism $\eta$ of the spectrum $Z$ of the center $\mathscr{Z}$ of $\mathscr{A}$ onto the spectrum of $Z_{1}$ of $\pi(\mathscr{Z})$ such that $\pi(A)^{\wedge}(\eta(\zeta))=A^{\wedge}(\zeta)$ for every $\zeta \in Z$. Let $\zeta$ be a fixed element in $Z$ and let $\eta(\zeta)=\zeta^{\prime}$. Then

$$
\phi(A)^{\wedge}(\zeta)=\tau_{E}(\pi(A))^{\wedge}\left(\zeta^{\prime}\right) .
$$

There is a homomorphism $\Psi$ of $\mathscr{B}$ with kernel [ $\left.\zeta^{\prime}\right]$ into the algebra of all bounded linear operators on the Hilbert space $H\left(\zeta^{\prime}\right)=\mathscr{B} E\left(\zeta^{\prime}\right)$. The ideal generated by the set of all abelian projections of $\mathscr{B}$ maps onto the set of all completely continuous operators of $H\left(\zeta^{\prime}\right)$ under $\Psi$. Let $x_{1}, x_{2}, \ldots, x_{m}$ be elements of $\mathscr{B} E\left(\zeta^{\prime}\right)$. There are elements $B_{1}, B_{2}, \ldots, B_{m}$ in $\mathscr{B}$ with $x_{j}=B_{j} E\left(\zeta^{\prime}\right)$ for $j=1,2, \ldots, m$. Let $B$ be an element in $\mathscr{B}$. There is an element $A$ in $\pi(\mathscr{A})$ such that $A B_{j} E=B B_{j} E$ for $j=1,2, \ldots, m$ (Theorem 4.3). This means $\Psi(A) x_{j}=\Psi(B) x_{j}$ for $j=1,2, \ldots, m$. This proves that $\Psi(\pi(\mathscr{A}))$ is irreducible on $H\left(\zeta^{\prime}\right)$. Let $x$ be the vector $E\left(\zeta^{\prime}\right)$ in $H\left(\zeta^{\prime}\right)$. We have that

$$
\phi(A)^{\wedge}(\zeta)=\tau_{E}(\pi(A))^{\wedge}\left(\zeta^{\prime}\right)=(\Psi(\pi(A)) x, x)
$$

for every $A$ in $\mathscr{A}$. This proves that $\phi(A)^{\wedge}(\zeta)$ is irreducible on $\mathscr{A}$. Q.E.D.

We now record some facts about the kernel of $\pi_{\phi}$.

Proposition 4.4. Let $\mathscr{A}$ be a von Neumann algebra and let $\phi$ be a state of the module $\mathscr{A}$. The kernel of $\pi_{\phi}$ is contained in the strong radical (viz, the intersection of all two-sided maximal ideals) of $\mathscr{A}$. In particular, if $\mathscr{A}$ is finite or if $\mathscr{A}$ is $\sigma$-finite and of type III, then $\pi_{\phi}$ is faithful.

Proof. Let $A$ be an element of $\mathscr{A}$. Let $\mathscr{K}_{A}^{\prime}$ be the uniform closure of the set

$$
\begin{aligned}
\left\{\sum\left\{\alpha_{i} U_{i}^{*} A U_{i} \mid i=1,2, \ldots, n\right\} \mid\right. & \alpha_{1}, \alpha_{2}, \ldots, \alpha_{n} \text { are positive of sum } 1 ; \\
& \left.U_{1}, U_{2}, \ldots, U_{n} \text { are unitary in } \mathscr{A} ; n=1,2, \ldots\right\} .
\end{aligned}
$$

Then $\mathscr{K}_{A}^{\prime} \cap \mathscr{Z}=\mathscr{K}_{A}$ is nonvoid for every $A$ in $\mathscr{A}$. If $\mathscr{A}$ is finite, then $\mathscr{K}_{A}$ contains a single element $A^{\#}$. In this case if $A \in \mathscr{A}^{+}$and $A^{\#}=0$, then $A=0$ [1, III, §5].

Assume first that $\mathscr{A}$ is finite. Set $\pi_{\phi}=\pi$ and let $A$ be an element of $\mathscr{A}$ such that $\pi(A)=0 ;$ then $\pi\left(A^{*} A\right)=0$. Since

$$
\pi\left(\sum \alpha_{i} U_{i}^{*} A^{*} A U_{i}\right)=\sum \alpha_{i} \pi\left(U_{i}^{*}\right) \pi\left(A^{*} A\right) \pi\left(U_{i}\right)=0
$$

and since $\pi$ is uniformly continuous, we have that $\pi\left(\left(A^{*} A\right)^{\#}\right)=0$. This means

$$
0=\phi\left(\left(A^{*} A\right)^{\#}\right)=\left(A^{*} A\right)^{\#} \text {. }
$$

Therefore, $A^{*} A=0$ and thus $\pi$ is faithful.

Now assume that $\mathscr{A}$ is properly infinite. The radical of $\mathscr{A}$ is the ideal of $\mathscr{A}$ all of 
whose positive elements $A$ satisfy the relation $\mathscr{K}_{A}=\{0\}$, [10, Proposition 2.4]. Therefore we readily conclude that $\pi(A)=0$ implies that $A$ is in the radical of $\mathscr{A}$.

Now in the general case there is a projection $P$ in the center of $\mathscr{A}$ such that $\mathscr{A} P$ is finite and $\mathscr{A}(1-P)$ is properly infinite. If $A$ is an element in the kernel of $\pi$, then $A P=0$ and $A(1-P)$ is in the radical of $\mathscr{A}(1-P)$. But the radical of $\mathscr{A}(1-P)$ is the radical of $\mathscr{A}$. So the kernel of $\pi$ is contained in the radical of $\mathscr{A}$. Q.E.D.

We now show that there are states which have faithful representations.

Proposition 4.5. Let $\mathscr{A}$ be a von Neumann algebra. There is a projection $E$ in $\mathscr{A}$ of central support 1 such that every state $\phi$ of the module $\mathscr{A}$ with the property $\phi(E)=1$ has a faithful representation $\pi_{\phi}$.

Proof. First let $\mathscr{A}$ be semifinite. Let $E$ be any finite projection of $\mathscr{A}$ of central support 1. Then let $\phi$ be a state of $\mathscr{A}$ such that $\phi(E)=1$. Let $F$ be a projection of $\mathscr{A}$ with $\pi(F)=0$ where $\pi=\pi_{\phi}$. Suppose $F$ has central support $P$. First assume that $F \leqq E P$. Since $E P$ is finite, there is a set $\left\{P_{i}\right\}$ of mutually orthogonal central projections of sum $P$ such that for each $P_{i}$ there is a set

$$
\left\{F_{i j} \mid 1 \leqq j \leqq n_{i}<+\infty\right\}
$$

of mutually orthogonal projections with the properties:

$$
F_{i j} \sim F P_{i} \quad \text { and } \quad F_{i}^{\prime}=E P_{i}-\sum_{j} F_{i j} \prec F P_{i} \quad[1, \mathrm{III}, \S 1] .
$$

Since $\pi\left(F P_{i}\right)=0$ we have that $\pi\left(F_{i j}\right)=0\left(1 \leqq j \leqq n_{i}\right)$ and $\pi\left(F_{i}^{\prime}\right)=0$. Indeed, if $V$ is a partial isometric operator and $\pi\left(V^{*} V\right)=0$, then $0=\pi\left(V^{*} V\right)=\pi(V)^{*} \pi(V)$ implies $\pi(V)=0$. So $\pi\left(V V^{*}\right)=0$. Then we conclude that $\pi\left(E P_{i}\right)=0$ for every $P_{i}$. This means $P_{i}=0$ and thus $P=0$. So $F=0$.

In the general case there is a central projection $P$ such that $F P \prec E P$ and $E(1-P)$ $\prec F(1-P)$. We have that $F P=0$ from the first part of the proof since we may assume $F P \leqq E P$. Also $\pi(E(1-P))=0$. So $1-P=0$. Thus, $F=0$.

Now let $A$ be any element of $\mathscr{A}$ such that $\pi(A)=0$. Suppose $\varepsilon>0$ is given; let $F_{1}, F_{2}, \ldots, F_{m}$ be orthogonal projections and let $\alpha_{1}, \alpha_{2}, \ldots, \alpha_{m}$ be positive numbers with $0 \leqq \sum \alpha_{i} F_{i} \leqq A^{*} A$ and $\left\|A^{*} A-\sum \alpha_{i} F_{i}\right\| \leqq \varepsilon$. Then $\pi\left(F_{i}\right)=0(i=1,2, \ldots, m)$ and so $F_{i}=0(i=1,2, \ldots, m)$. We obtain this from the first part. This shows $\left\|A^{*} A\right\| \leqq \varepsilon$. Since $\varepsilon>0$ is arbitrary, we have that $A=0$. This shows that $\pi$ is faithful if $\mathscr{A}$ is semifinite.

Now let $\mathscr{A}$ be a purely infinite von Neumann algebra with no nonzero $\sigma$-finite central projections. There is a net $\left\{P_{i}\right\}$ of orthogonal central projections of sum 1 such that each $P_{i}$ is least upper bound of a set $S_{i}$ of equivalent mutually orthogonal $\sigma$-finite projections [1, III, $\S 1$, Lemma 7]. For each $i$ let $E_{i} \in S_{i}$ and let $E=\sum E_{i}$. Then $E$ is a projection of central support 1 . If $F$ is a projection of central support $Q$ then $E Q P_{i} \prec F P_{i}$ for each $P_{i}[1, \mathrm{III}, \S 8$, Corollary 5]. So $E Q \prec F$.

Let $\phi$ be a state of the module $\mathscr{A}$ such that $\phi(E)=1$. We show that the kernel of $\pi_{\phi}=\pi$ is 0 . It is sufficient to show that $\pi(F)=0$ implies $F=0$ whenever $F$ is a pro- 
jection. However, if $F$ has central support $Q$ then $E Q \prec F$. So $\pi(E Q)=0$ and thus $\phi(E Q)=Q=0$. This proves $F=0$.

Now let $\mathscr{A}$ be a purely infinite algebra. There is a projection $P$ of $\mathscr{A}$ such that $P$ is the least upper bound of $\sigma$-finite central projections and such that $1-P$ majorizes no nonzero $\sigma$-finite central projections. Now let $F$ be any projection in $\mathscr{A} P$ of central support 1 and let $E$ be a projection previously constructed for a purely infinite von Neumann algebra with no nonzero $\sigma$-finite central projections. Let $\phi$ be a state of $\mathscr{A}$ such that $\phi(E+F)=1$. The canonical representation $\pi$ induced by $\phi$ has kernel equal to (0) [Proposition 4.4].

The general result for an arbitrary von Neumann $\mathscr{A}$ algebra now follows from the fact that there is a central projection $P$ such that $\mathscr{A} P$ is semifinite and $\mathscr{A}(1-P)$ is purely infinite. Q.E.D.

THEOREM 4.6. Let $\mathscr{A}$ be a von Neumann algebra. There is a pure state of the module $\mathscr{A}$ whose canonical representation is faithful.

Proof. There is a projection $E$ of $\mathscr{A}$ of central support 1 such that the canonical representation $\pi_{\phi}$ induced by a state $\phi$ of the module $\mathscr{A}$ is faithful whenever $\phi(E)=1$ (Proposition 4.5). By Proposition 4.1 there is a pure state $\phi$ of the module $\mathscr{A}$ such that $\phi(E)=1$. Q.E.D.

THEOREM 4.7. Let $\mathscr{A}$ be a von Neumann algebra and let $\zeta$ be a maximal ideal of the center of $\mathscr{A}$. The smallest closed two-sided ideal $[\zeta]$ in $\mathscr{A}$ containing $\zeta$ is a primitive ideal.

Proof. Let $\phi$ be a pure state of $\mathscr{A}$ whose canonical representation $\pi_{\phi}$ is faithful. The representation $\pi$ of $\mathscr{A}$ on the space $H$ satisfying properties (1)-(5) of Proposition 4.2 constructed relative to $\phi$ is faithful. Let $\zeta^{\prime}=\pi(\zeta)$ and let [ $\left.\zeta^{\prime}\right]$ be the smallest closed two-sided ideal in the von Neumann algebra $\mathscr{B}$ generated by $\pi(\mathscr{A})$ on $H$ which contains $\zeta^{\prime}$. There is an irreducible representation $\Psi$ of $\pi(\mathscr{A})$ with kernel $\pi(\mathscr{A}) \cap\left[\zeta^{\prime}\right]$ (corollary, Theorem 4.3). However $\pi(\mathscr{A}) \cap\left[\zeta^{\prime}\right]$ is the smallest closed two-sided ideal $J$ of $\pi(\mathscr{A})$ which contains $\zeta^{\prime}$. Indeed, if $E$ is a projection in $\pi(\mathscr{A})$ $\cap\left[\zeta^{\prime}\right]$ then the Gelfand transform $P^{\wedge}$ of the central support $P$ of $E$ vanishes at the point $\zeta^{\prime}$. Thus the projection $P$ is in the maximal ideal $\zeta^{\prime}$ and so $E$ is in the ideal $J$. Because $J$ contains all projections of $\pi(\mathscr{A}) \cap\left[\zeta^{\prime}\right]$, the ideal $\pi(\mathscr{A}) \cap\left[\zeta^{\prime}\right]$ is contained in $J$. Therefore we have that $J=\pi(\mathscr{A}) \cap\left[\zeta^{\prime}\right]$. However, $\pi^{-1}(J)=[\zeta]$ since $\pi$ is

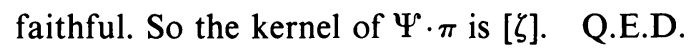

The set Prim $(\mathscr{A})$ of all primitive ideals of $\mathscr{A}$ supplied with the hull-kernel topology is called structure space of $\mathscr{A}$.

Proposition 4.7. Let $\mathscr{A}$ be a von Neumann algebra with center $\mathscr{Z}$. Let $Z$ be the spectrum of $\mathscr{Z}$. The set $\{[\zeta] \mid \zeta \in Z\}$ is dense in the structure space of $\mathscr{A}$.

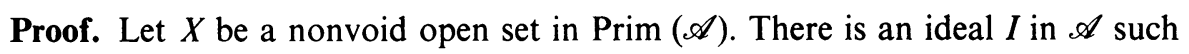
that

$$
X=\{J \in \operatorname{Prim}(\mathscr{A}) \mid J \neq I\} .
$$


Let $J$ be an ideal in $X$ and let $J \cap \mathscr{Z}=\zeta$. The ideal $\zeta$ is maximal in $\mathscr{Z}$. We have that $[\zeta] \ngtr I$ since $[\zeta] \subset J$. This proves that $[\zeta] \in X$. Thus $\{[\zeta] \mid \zeta \in Z\}$ is dense in Prim $(\mathscr{A})$. Q.E.D.

The set $\mathscr{A}^{\wedge}$ of unitary equivalence classes of irreducible representations of $\mathscr{A}$ with the topology induced by the map $\pi \rightarrow \operatorname{kernel} \pi$ of $\mathscr{A}^{\wedge}$ into Prim $(\mathscr{A})$ is known to be a Baire space $[2,3.4 .13]$. A proof of this fact is obtainable from the preceding proposition.

The next theorem characterizes a pure state in terms of its kernel. It is the analogue of a theorem of Kadison [13].

THEOREM 4.8. Let $\mathscr{A}$ be a von Neumann algebra. A state $\phi$ of the module $\mathscr{A}$ is a pure state if and only if the kernel of $\phi$ is the sum of the sets

$$
L_{\phi}=\left\{A \in \mathscr{A} \mid \phi\left(A^{*} A\right)=0\right\} \text { and } L_{\phi}^{*}=\left\{A \in \mathscr{A} \mid A^{*} \in L_{\phi}\right\} .
$$

Proof. Suppose $\phi$ is a pure state of the module $\mathscr{A}$. Let $\pi$ be a representation of $\mathscr{A}$ on a Hilbert space $H$ which satisfies properties (1)-(5) of Proposition 4.2 with respect to $\phi$. Let $E$ be the abelian projection of the von Neumann algebra $\mathscr{B}$ generated by $\pi(\mathscr{A})$ such that $\pi(\phi(A))=\tau_{E}(\pi(A))$ for every $A$ in $\mathscr{A}$. Suppose $A$ is a point of the kernel of $\phi$. The range projection $F$ of $\pi(A) E$ in $\mathscr{B}$ is an abelian projection orthogonal to $E$. There is a hermitian element $C$ in $\mathscr{A}$ such that $\pi(C) \pi(A) E$ $=\pi(A) E$ and $\pi(C) E=0$ (Theorem 4.3). Thus, $A-C A \in L_{\phi}$ and $A^{*} C \in L_{\phi}$. So $A=(A-C A)+C A$ is an element of $L_{\phi}+L_{\phi}^{*}$. This proves that $L_{\phi}+L_{\phi}^{*}$ contains the kernel of $\phi$. Because $|\phi(A)|^{2} \leqq \phi\left(A^{*} A\right)$ for every $A$ in $\mathscr{A}$, the kernel of $\phi$ contains $L_{\phi}+L_{\phi}^{*}$. So the kernel of $\phi$ is equal to $L_{\phi}+L_{\phi}^{*}$.

Conversely, let $L_{\phi}+L_{\phi}^{*}$ be the kernel of $\phi$. Let $C$ be a central element of $\mathscr{A}$ strictly between 0 and 1 and let $\phi_{1}$ and $\phi_{2}$ be two positive functionals of the module $\mathscr{A}$ of norm not exceeding 1 such that $C \phi_{1}+(1-C) \phi_{2}=\phi$. First notice that $\phi_{1}$ and $\phi_{2}$ are states of $\mathscr{A}$. Then if $\phi(A)=0$, there are elements $B_{1}$ and $B_{2}$ in $L_{\phi}$ and $L_{\phi}^{*}$ respectively such that $A=B_{1}+B_{2}$. Because $\phi\left(B_{1}^{*} B_{1}\right)=\phi\left(B_{2} B_{2}^{*}\right)=0$, we have that $\phi_{1}\left(B_{j}\right)=\phi_{2}\left(B_{j}\right)=0$ for $j=1,2$. Thus $\phi_{1}(A)=\phi_{2}(A)=0$. Now for arbitrary $A$ in $\mathscr{A}$ there is a central element $B$ in $\mathscr{A}$ such that $\phi(A-B)=0$. Thus $\phi_{1}(A-B)=\phi_{2}(A-B)$ $=0$ and so $\phi_{1}(A)=\phi_{2}(A)=B=\phi(A)$. This proves $\phi$ is a pure state. Q.E.D.

5. Pointwise convergence of states. Let $\mathscr{A}$ be a von Neumann algebra. A net of states $\left\{\phi_{n}\right\}$ of the module $\mathscr{A}$ is said to converge pointwise to a state $\phi$ if $\left\{\phi_{n}(A)\right\}$ converges uniformly to $\phi(A)$ for every $A$ in $\mathscr{A}$. The set $E(\mathscr{A})$ of states of the module $\mathscr{A}$ taken with the topology of pointwise convergence is called the state space of $\mathscr{A}$. The closure in the state space of the module $\mathscr{A}$ of the set of pure states in $\mathscr{A}$ is called the pure state space of the module $\mathscr{A}$. It is denoted by $P(\mathscr{A})$. An element $\phi$ in $E(\mathscr{A})$ is said to be a vector state if there is an abelian projection $E$ in the commutator of the center of $\mathscr{A}$ such that $\phi(A)=\tau_{E}(A)$ for every $A$ in $\mathscr{A}$. The closure in the space $E(\mathscr{A})$ of the set of vector states is called the vector state space of $\mathscr{A}$. It is denoted by $V(\mathscr{A})$. 
We now study the structure of $P(\mathscr{A})$ and $V(\mathscr{A})$ using the theorems of Glimm $[3, \S 4]$ as our guide.

THEOREM 5.1. If $\mathscr{A}$ is a continuous von Neumann algebra, the state space, the pure state space, and the vector state space of the module $\mathscr{A}$ coincide.

Proof. First we show that the vector state space $V(\mathscr{A})$ of the module $\mathscr{A}$ coincides with the state space $E(\mathscr{A})$ of the module $\mathscr{A}$. Let $\phi$ be an element of $E(\mathscr{A})$ and let $A_{1}, A_{2}, \ldots, A_{n}$ be elements of $\mathscr{A}$. Assume $A_{1}=1$. Let $\mathscr{Z}^{\prime}$ be the commutator of the

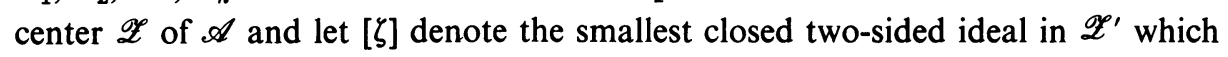
contains the maximal ideal $\zeta$ of $\mathscr{Z}$. There is for each ideal $\zeta$ an irreducible representation $\Psi_{\zeta}$ of $\mathscr{Z}^{\prime}$ with kernel [ $\zeta$ ] on the algebra of bounded linear operators of a Hilbert space $H(\zeta)$ such that $\Psi_{\zeta}\left(\mathscr{Z}^{\prime}\right)$ contains the ideal $C(H(\zeta))$ of completely continuous operators on $H(\zeta)$. Since $\mathscr{A}$ is a continuous algebra, the image $\Psi_{\zeta}(\mathscr{A})$ of $\mathscr{A}$ contains no minimal projections. So $\Psi_{\zeta}(\mathscr{A}) \cap C(H(\zeta))=(0)$. There is unit vector $x_{\zeta}$ in $H(\zeta)$ such that

$$
\left|\phi\left(A_{j}\right)^{\wedge}(\zeta)-\left(\Psi_{\zeta}\left(A_{j}\right) x_{\zeta}, x_{\zeta}\right)\right|<\frac{1}{2} \text { for } j=1,2, \ldots, n .
$$

Indeed, the kernel of the functional $A \rightarrow \phi(A)^{\wedge}(\zeta)$ of $\mathscr{A}$ contains the ideal $\mathscr{A} \cap[\zeta]$. So there is a functional $\phi_{\zeta}$ of $\Psi_{\zeta}(\mathscr{A})$ such that

$$
\phi_{\zeta} \cdot \Psi_{\zeta}(A)=\phi(A)^{\wedge}(\zeta)
$$

Then the statement in question simply states that the functional $\phi_{\zeta}$ is the pointwise limit of vector states of $\Psi_{\zeta}(\mathscr{A})[2,11.2 .1]$. There is an abelian projection $E_{\zeta}$ of $\mathscr{Z}^{\prime}$ such that

$$
\left(\Psi_{\zeta}(B) x_{\zeta}, x_{\zeta}\right)=\tau_{E_{\zeta}}(B)^{\wedge}(\zeta)
$$

for every $B$ in $\mathscr{Z}^{\prime}$ (cf. [7, Theorem 1]). This means that there is a central projection $P$ with $P^{\wedge}(\zeta)=1$ such that $E_{\zeta} P$ has central support $P$ and such that

$$
\left\|\phi\left(A_{j}\right) P-\tau_{E_{\zeta} P}\left(A_{j}\right)\right\|<1
$$

for $j=1,2, \ldots, n$. Thus there is an abelian projection $E$ of central support 1 such that $\left\|\phi\left(A_{j}\right)-\tau_{E}\left(A_{j}\right)\right\|<1$ for $j=1,2, \ldots, n$. This shows that $\phi$ is the pointwise limit of vector states. Thus $E(\mathscr{A})=V(\mathscr{A})$.

We now show that $E(\mathscr{A})$ is equal to the pure state space of the module $\mathscr{A}$. First let $\psi$ be any pure state of the module $\mathscr{A}$ whose canonical representation $\pi_{\psi}$ is faithful (Theorem 4.6). Let $\pi$ be a faithful representation of $\mathscr{A}$ on a Hilbert space $H$ such that the commutator $\pi(\mathscr{A})^{\prime}$ of $\pi(\mathscr{A})$ on $H$ is equal to $\pi(\mathscr{Z})$ and such that there is an abelian projection $E$ in $\pi(\mathscr{Z})^{\prime}$ of central support 1 with the property $\tau_{E}(\pi(A))$ $=\pi(\psi(A))$ for every $A$ in $\mathscr{A}$ (Proposition 4.2). Let $\phi$ be an element of $E(\mathscr{A})$ and let $A_{1}, A_{2}, \ldots, A_{m}$ be elements of $\mathscr{A}$. There is an abelian projection $F$ of central support 1 in $\pi(\mathscr{Z})^{\prime}$ such that

$$
\left\|\pi \cdot \phi \cdot \pi^{-1}\left(\pi\left(A_{j}\right)\right)-\tau_{F}\left(\pi\left(A_{j}\right)\right)\right\|<1
$$


for $j=1,2, \ldots, m$. Indeed, if $\zeta$ is a maximal ideal in $\pi(\mathscr{Z})$, there is an irreducible

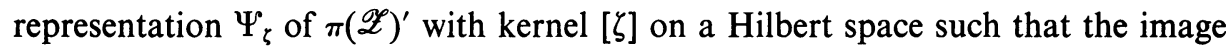
of $\pi(\mathscr{A})$ contains no completely continuous operators. The same reasoning as the previous paragraph therefore is applicable. So it is sufficient to show that $\pi^{-1} \cdot \tau_{F} \cdot \pi$ is a pure state of $\mathscr{A}$. We do this by showing that it is $\mathscr{Z}$-irreducible. Let $\theta$ be a positive functional of the module $\mathscr{A}$ majorized by $\pi^{-1} \cdot \tau_{F} \cdot \pi$. Then $\theta^{\prime}=\pi \cdot \theta \cdot \pi^{-1}$ on $\pi(\mathscr{A})$ is majorized by $\tau_{F}$ on $\pi(\mathscr{A})$. Let $\zeta$ be a maximal ideal in $\pi(\mathscr{Z})$. There are positive functionals $f$ and $g$ on $\Psi_{\zeta}(\pi(\mathscr{A}))$ such that

$$
f\left(\Psi_{\zeta}(A)\right)=\theta^{\prime}(A)^{\wedge}(\zeta) \text { and } g\left(\Psi_{\zeta}(A)\right)=\tau_{F}(A)^{\wedge}(\zeta)
$$

for $A$ in $\pi(\mathscr{A})$. Then $g$ majorizes $f$ on $\Psi_{\zeta}(\pi(\mathscr{A}))$. However $g$ is irreducible on $\Psi_{\zeta}(\pi(\mathscr{A}))$ and so there is an $\alpha_{\zeta}$ in the complex field such that $f(A)=\alpha_{\zeta} g(A)$ for all $A$ in $\Psi_{\zeta}(\pi(\mathscr{A}))$. But $\alpha_{\zeta}=\theta^{\prime}(1)^{\wedge}(\zeta)$. Since $\zeta$ is arbitrary we have that $\theta^{\prime}=\theta^{\prime}(1) \tau_{F}$ on $\pi(\mathscr{A})$. This proves that $\pi^{-1} \cdot \tau_{F} \cdot \pi$ is $\mathscr{Z}$-irreducible. Q.E.D.

We see that if $\pi$ is a faithful representation of the continuous algebra $\mathscr{A}$ on a Hilbert space $H$ with the property that the commutator of $\pi(\mathscr{A})$ is $\pi(\mathscr{Z})$ and that there is an abelian projection $E$ with central support 1 in the commutator $\pi(\mathscr{Z})^{\prime}$ of $\pi(\mathscr{Z})$ such that $\pi^{-1} \cdot \tau_{E} \cdot \pi$ is a pure state of $\mathscr{A}$, then the set

$$
\left\{\pi^{-1} \cdot \tau_{F} \cdot \pi \mid F \text { is an abelian projection of central support } 1 \text { in } \pi(\mathscr{Z})^{\prime}\right\}
$$

is pointwise dense in $E(\mathscr{A})$.

We now identify the pure state and vector state spaces of a type I algebra. We begin with the following theorem.

THEOREM 5.2. If $\mathscr{A}$ is a type I von Neumann algebra, the vector state space $V(\mathscr{A})$ of the module $\mathscr{A}$ is equal to the pure state space $P(\mathscr{A})$ of the module $\mathscr{A}$.

Proof. Since every vector state of the module $\mathscr{A}$ is a pure state of the module $\mathscr{A}$, we have that $V(\mathscr{A}) \subset P(\mathscr{A})[12$, Remark, Theorem 9].

Now let $\phi$ be a pure state of the module $\mathscr{A}$. Let $A_{1}, A_{2}, \ldots, A_{m}$ be elements of $\mathscr{A}$. For each maximal ideal $\zeta$ of the center of $\mathscr{A}$ there is an irreducible representation

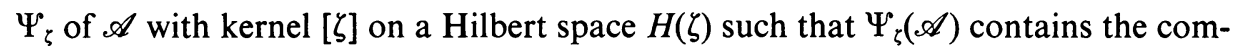
pletely continuous operators on $H(\zeta)$. The kernel of the function $A \rightarrow \phi(A)^{\wedge}(\zeta)$ on

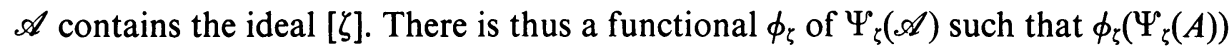
$=\phi(A)^{\wedge}(\zeta)$ for every $A$. Since $\phi(A)^{\wedge}(\zeta)$ is a pure state of $\mathscr{A}$ (corollary, Theorem 4.3), the functional $\phi_{\zeta}$ is a pure state of $\Psi_{\zeta}(\mathscr{A})$. The pure state space of $\Psi_{\zeta}(\mathscr{A})$ is equal to the vector space of $\Psi_{\zeta}(\mathscr{A})$ (cf. $[2,3.4 .1]$ due to R. V. Kadison). There is a unit vector $x_{\zeta}$ in $H(\zeta)$ such that

$$
\left|\phi_{\zeta}\left(\Psi_{\zeta}\left(A_{j}\right)\right)-\left(\Psi_{\zeta}\left(A_{j}\right) x_{\zeta}, x_{\zeta}\right)\right|<1
$$

for $j=1,2, \ldots, m$. There is an abelian projection $E_{\zeta}$ in $\mathscr{A}$ such that

$$
\left(\Psi_{\zeta}(A) x_{\zeta}, x_{\zeta}\right)=\tau_{E_{\zeta}}(A)^{\wedge}(\zeta)
$$


for every $A$ in $\mathscr{A}$. By the same reasoning as Theorem 5.1 we obtain an abelian projection $E$ in $\mathscr{A}$ of central support 1 such that

$$
\left\|\phi\left(A_{j}\right)-\tau_{E}\left(A_{j}\right)\right\|<1
$$

for $j=1,2, \ldots, m$. This means that $\phi \in V(\mathscr{A})$. Therefore, $P(\mathscr{A}) \subset V(\mathscr{A})$. This completes the proof.

Let $\mathscr{A}$ be a type I von Neumann algebra with center $\mathscr{Z}$. The uniformly closed *-subalgebra of $\mathscr{A}$ generated by the abelian projections of $\mathscr{A}$ is a two-sided ideal $I_{a}$ in $\mathscr{A}$ [16]. If $A \in I_{a}^{+}$, there is a sequence $\left\{A_{n}\right\}$ of positive central elements and a sequence $\left\{E_{n}\right\}$ of orthogonal abelian projections such that

(1) $A_{1} \geqq A_{2} \geqq \cdots$;

(2) $\lim A_{n}=0$ (uniformly);

(3) the central support of $E_{n}$ has Gelfand transform equal to the characteristic function of the support for the Gelfand transform of $A_{n}$ for each $n=1,2, \ldots$;

(4) $A=\sum A_{n} E_{n}$; and

(5) the sequence $\left\{A_{n}\right\}$ is uniquely determined.

The sum $\sum A_{n} E_{n}$ is called a spectral decomposition of $A$.

Let $\mathscr{T}$ be the trace class of $\mathscr{A}$ and let $\operatorname{Tr}$ be the canonical trace of $\mathscr{A}(\S 4)$. For each $A$ in $\mathscr{T}$ define the bounded module homomorphism $\Phi_{A}$ of $I_{a}$ into $\mathscr{Z}$ by $\Phi_{A}(B)=\operatorname{Tr}(A B)$. Then if $\mathscr{T}$ is given the norm

$$
\|A\|_{1}=\left\|\operatorname{Tr}\left(\left(A^{*} A\right)^{1 / 2}\right)\right\|,
$$

the function $A \rightarrow \Phi_{A}$ defines an order preserving isometric isomorphism of the $\mathscr{Z}$-module $\mathscr{T}$ onto the set of all bounded module homomorphisms of $I_{a}$ into $\mathscr{Z}$ $[9, \S 4]$.

THEOREM 5.3. Let $\mathscr{A}$ be a type I von Neumann algebra. Let $I_{a}$ be the closed twosided ideal of $\mathscr{A}$ generated by the abelian projections of $\mathscr{A}$. The vector state space $V(\mathscr{A})$ of the module $\mathscr{A}$ consists of the set of all states of the module $\mathscr{A}$ of the form

$$
C \phi+(1-C) \tau_{E}
$$

where $C$ is a central element of $\mathscr{A}$ with $0 \leqq C \leqq 1, \psi$ is a state of the module $\mathscr{A}$ such that $C \psi$ vanishes on $I_{a}$ and $E$ is a maximal abelian projection of $\mathscr{A}$.

Proof. First let $\phi$ be an element of $V(\mathscr{A})$; set $\phi \mid I_{a}=\theta_{1}$. There is a positive element $B$ in the trace class of $\mathscr{A}$ such that $\theta_{1}(A)=\operatorname{Tr}(A B)$ for every $A$ in $I_{a}$. Let $\theta(A)=\operatorname{Tr}(A B)$ for every $A$ in $\mathscr{A}$. We show that the functional $\phi-\theta$ is positive. Let $A \in \mathscr{A}^{+}$. There is a monotonely increasing net $\left\{A_{n}\right\}$ in $I_{a}^{+}$which converges strongly to $A\left[1, \mathrm{I}, \S 3\right.$, Theorem 2, Corollary 5] because $I_{a}$ is strongly dense in $\mathscr{A}$. Let $x$ be a vector in the Hilbert space of $\mathscr{A}$. We have that

$$
(\phi(A) x, x)-\left(\theta\left(A_{n}\right) x, x\right) \geqq\left(\phi\left(A_{n}\right) x, x\right)-\left(\theta\left(A_{n}\right) x, x\right)=0
$$

for every $A_{n}$. Thus

$$
(\phi(A) x, x)-(\theta(A) x, x)=\lim _{n}\left((\phi(A) x, x)-\left(\theta\left(A_{n}\right) x, x\right)\right) \geqq 0 .
$$


This proves $\phi-\theta$ is a positive functional of the module $\mathscr{A}$. We also have that $\phi(A)-\theta(A)=0$ for every $A \in I_{a}$.

Now let $B=\sum B_{i} E_{i}$ be a spectral decomposition for $B$. Here $\left\{E_{i}\right\}$ is a sequence of orthogonal abelian projections with $E_{1} \succ E_{2} \succ \cdots ;\left\{B_{i}\right\}$ is a decreasing sequence of positive central elements with $\lim B_{n}=0$ (uniformly); and the support of each $B_{i}$ is equal to the central support of $E_{i}$. There is a set of mutually orthogonal central projections $\left\{P_{n}\right\}$ of sum 1 such that for each $P_{n}$ the series $\sum\left\{P_{n} B_{i} \mid i=1,2, \ldots\right\}$ converges uniformly [9, Theorem 4.1]. Let $n$ be fixed and let $X_{n}$ be the set of $\zeta$ in the spectrum $Z$ of the center of $\mathscr{A}$ such that $P_{n}(\zeta)=1$. For $\zeta \in X_{n}$ let $\Psi_{\zeta}$ be an irreducible

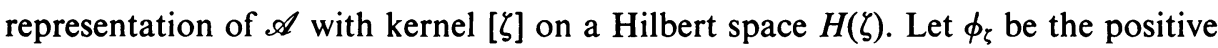
functional on $\Psi_{\zeta}(\mathscr{A})=\mathscr{A}(\zeta)$ given by $\phi_{\zeta}(A(\zeta))=\phi(A)^{\wedge}(\zeta)$. Here $\Psi_{\zeta}(A)=A(\zeta)$. Since every functional $f$ having the form $f(A(\zeta))=\tau_{F}(A)^{\wedge}(\zeta)$, where $F$ is an abelian projection of $\mathscr{A}$ of central support 1 , is a vector state of $\mathscr{A}(\zeta)$, the functional $\phi_{\zeta}$ is in the vector state space of $\mathscr{A}(\zeta)$. By Glimm's theorem [3, Theorem 2], there is an $\alpha_{\zeta}$ in the interval $[0,1]$, a state $g_{\zeta}$ of $\mathscr{A}(\zeta)$ vanishing on the completely continuous operators of $H(\zeta)$, and a unit vector $x_{\zeta}$ in $H(\zeta)$ such that

$$
\phi_{\zeta}=\alpha_{\zeta} g_{\zeta}+\left(1-\alpha_{\zeta}\right) w_{x_{\zeta}} .
$$

Now we have that

$$
\theta(A)^{\wedge}(\zeta)=\left(\sum B_{i} \tau_{E_{i}}(A)\right)^{\wedge}(\zeta)=\sum B_{i}^{\wedge}(\zeta) \tau_{E_{i}}(A)^{\wedge}(\zeta)
$$

by the uniform convergence of $\sum_{i} B_{i} P_{n}$. Since $\Psi_{\zeta}\left(I_{a}\right)$ is precisely the ideal of completely continuous operators on $H(\zeta)$, we must have that

$$
\left(1-\alpha_{\zeta}\right) w_{x_{\zeta}}(A(\zeta))=\sum B_{i}(\zeta) \tau_{E_{i}}(A)^{\wedge}(\zeta)
$$

for each $A$ in $I_{a}$. For each $E_{i}$ there is a unit vector $y_{i}$ in $H(\zeta)$ such that

$$
B_{i}^{\wedge}(\zeta) \tau_{E_{i}}(A)^{\wedge}(\zeta)=B_{i}^{\wedge}(\zeta)\left(A(\zeta) y_{i}, y_{i}\right) .
$$

Indeed, $E_{i}(\zeta)$ is a projection on $H(\zeta)$ of dimension not exceeding 1 . Therefore, we have that

$$
\left(1-\alpha_{\zeta}\right) w_{x_{\zeta}}(A(\zeta))=B_{1}^{\wedge}(\zeta) \tau_{E_{1}}(A)^{\wedge}(\zeta)
$$

for every $A$ in $I_{a}$. Then $B_{2}(\zeta), B_{3}(\zeta), \ldots$ vanish. Because $\zeta$ in $X_{n}$ is arbitrary, we conclude that $0=B_{2} P_{n}=B_{3} P_{n}=\cdots$ and thus that $B P_{n}=\left(B_{1} E_{1}\right) P_{n}$. Because $P_{n}$ is arbitrary, we find that $B_{2}, B_{3}, \ldots$ vanish. Thus $B=B_{1} E_{1}$ and $\theta(A)=B_{1} \tau_{E_{1}}(A)$ for every $A$ in $\mathscr{A}$. Since the support of $B_{1}^{\wedge}$ is equal to that of $E_{1}$, we may assume $E=E_{1}$ is a maximal abelian projection and still retain the formula $B_{1} \tau_{E}(A)=\theta(A)$.

There is a sequence $\left\{Q_{n}\right\}$ of orthogonal central projections of sum equal to the support $Q$ of $C=\phi(1)-\theta(1)$ such that for each $Q_{n}$ there is a positive central element $D_{n}$ with $D_{n} Q_{n}=D_{n}$ and $D_{n} C=Q_{n}$. The sequence $\left\{\left\|D_{n}(\phi(A)-\theta(A))\right\|\right\}$ is bounded above by $\|A\|$ for each $A$ in $\mathscr{A}$ since $\phi-\theta$ is a positive functional of the module $\mathscr{A}$. Set $\psi_{1}(A)=\sum_{n} D_{n}(\phi(A)-\theta(A))$ for each $A$ in $\mathscr{A}$. Then $\psi$ is a positive 
functional of the module $\mathscr{A}$, with the property $\psi_{1}(1)=Q$. We extend $\psi_{1}$ to a state $\psi$ on the module $\mathscr{A}$ by setting $\psi=\psi_{1}+\psi_{2}$ where $\psi_{2}$ is a positive functional of the module $\mathscr{A}$ with $\psi_{2}(1)=1-Q$.

We show that $C \psi+B_{1} \tau_{E}=\phi$. For each $Q_{n}$ we have that

$$
Q_{n}\left(C \psi(A)+B_{1} \tau_{E}(A)\right)=Q_{n}(\phi(A)-\theta(A)+\theta(A))=Q_{n} \phi(A)
$$

for every $A$ in $\mathscr{A}$. Also

$$
(1-Q)\left(C \psi(A)+B_{1} \tau_{E}(A)\right)=(1-Q) \theta(A)=(1-Q) \phi(A) .
$$

So $C \psi+B_{1} \tau_{E}=\phi$. Since both $\psi$ and $\tau_{E}$ are states, we have that $C+B_{1}=1$. This completes the first part of the proof.

Conversely, let $\phi$ be a state of the module $\mathscr{A}$ of the form

$$
\phi=C \psi+(1-C) \tau_{E},
$$

where $C$ is a central element of $\mathscr{A}$ with $0 \leqq C \leqq 1, \psi$ is a state of the module $\mathscr{A}$ such that $C \psi$ vanishes on $I_{a}$, and $E$ is an abelian projection of central support 1 . Let $A_{1}, A_{2}, \ldots, A_{n}$ be elements of $\mathscr{A}$. Let $\zeta$ be a maximal ideal of the center of $\mathscr{A}$ and

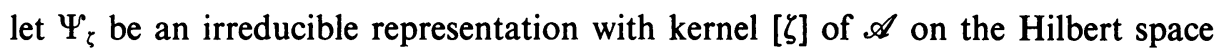
$H(\zeta)$. Let $\Psi_{\zeta}(\mathscr{A})=\mathscr{A}(\zeta)$ and $\Psi_{\zeta}(A)=A(\zeta)$. The relation

$$
\phi_{\zeta}(A(\zeta))=\phi(A) \wedge(\zeta)
$$

defines a functional in the vector state space of $\mathscr{A}(\zeta)$ [3, Theorem 2] since $\Psi_{\zeta}\left(I_{a}\right)$ is the ideal of completely continuous operators on $H(\zeta)$. There is a unit vector $x_{\zeta}$ in $H(\zeta)$ such that

$$
\left|\phi_{\zeta}\left(A_{j}(\zeta)\right)-\left(A_{j}(\zeta) x_{\zeta}, x_{\zeta}\right)\right|<1
$$

for $j=1,2, \ldots, n$. But there is an abelian projection $E_{\zeta}$ in $A$ such that

$$
\left(A(\zeta) x_{\zeta}, x_{\zeta}\right)=\tau_{E_{\zeta}}(A)^{\wedge}(\zeta)
$$

for every $A$ in $\mathscr{A}$. By the same procedure as employed in Theorem 5.2, we obtain an abelian projection $F$ of central support 1 in $\mathscr{A}$ such that

$$
\left|\phi_{\zeta}\left(A_{j}(\zeta)\right)-\tau_{F}\left(A_{j}\right)^{\wedge}(\zeta)\right|<1
$$

for every $j=1,2, \ldots, n$ and every maximal ideal $\zeta$. So

$$
\left\|\phi\left(A_{j}\right)-\tau_{F}\left(A_{j}\right)\right\|<1
$$

for $j=1,2, \ldots, n$. Thus $\phi$ is in the vector state space of the module $\mathscr{A}$. Q.E.D.

In a type I algebra every state is the pointwise limit of $\sigma$-weakly continuous states.

THEOREM 5.4. Let $\mathscr{A}$ be a type I von Neumann algebra. Every state of the module $\mathscr{A}$ is the pointwise limit of normal states of the module $\mathscr{A}$.

Proof. Let $\phi$ be a state of $\mathscr{A}$. Let $\theta_{1}$ be the restriction of $\phi$ to $I_{a}$. There is a positive element $B$ of the trace class of $\mathscr{A}$ such that $\theta_{1}(A)=\operatorname{Tr}(B A)$ for every $A$ in $I_{a}$. Let 
$\theta(A)=\operatorname{Tr}(B A)$ for every $A$ in $\mathscr{A}$. Then $\phi-\theta=\psi_{1}$ is a positive functional on the module $\mathscr{A}$ which vanishes on $I_{a}$ (cf. proof of Theorem 5.3). Let the central projection $Q$ be the support of $C=\psi_{1}(1)$. There is a positive functional $\psi$ of the module $\mathscr{A}$ such that $\psi(1)=Q$ and such that $C \psi=\psi_{1}$. Now let $A_{1}, A_{2}, \ldots, A_{n}$ be elements of $\mathscr{A}$. The restriction of $\psi$ to the $\mathscr{Z} Q$-module $\mathscr{A} Q$ vanishes on the closed two-sided ideal $I_{a} Q$ generated by the abelian projections of $\mathscr{A} Q$. There is an abelian projection $E$ in $\mathscr{A} Q$ with central support $Q$ such that

$$
\left\|\psi(A Q)-\tau_{E}\left(A_{j} Q\right)\right\|<(\|C\|+1)^{-1}
$$

for $j=1,2, \ldots, n$ (Theorem 5.3). This means that

$$
\left\|\psi_{1}\left(A_{j}\right)-C \tau_{E}\left(A_{j}\right)\right\|<1
$$

for $j=1,2, \ldots, n$. The functional

$$
A \rightarrow C \tau_{E}(A)+\operatorname{Tr}(B A)
$$

is a $\sigma$-weakly continuous positive functional of the module $\mathscr{A}$. We have that

$$
C \tau_{E}(1)+\operatorname{Tr}(B)=\phi(1)-\theta(1)+\theta(1)=\phi(1)=1 .
$$

Also

$$
\left\|\phi\left(A_{j}\right)-C \tau_{E}\left(A_{j}\right)-\operatorname{Tr}\left(B A_{j}\right)\right\|<1
$$

for $j=1,2, \ldots, n$. Thus the state $\phi$ is the pointwise limit of positive $\sigma$-weakly continuous states. Q.E.D.

\section{BIBLIOGRAPHY}

1. J. Dixmier, Les algèbres d'opérateurs dans l'espace hilbertien, Gauthier-Villars, Paris, 1957.

2. - Les $C^{*}$-algèbres et leur représentations, Gauthier-Villars, Paris, 1964.

3. J. Glimm, A Stone-Weierstrass theorem for $C^{*}$-algebras, Ann. of Math. (2) 72 (1960), 216-244.

4. —- Type I C*-algebras, Ann. of Math. (2) 73 (1961), 572-612.

5. R. Godement, Sur la théorie des représentations unitaires, Ann. of Math. (2) 53 (1951), 68-124.

6. M. Goldman, Structure of $A W^{*}$-algebras of type I, Duke Math. J. 23 (1956), 23-34.

7. H. Halpern, The maximal GCR ideal of an $A W^{*}$-algebra, Proc. Amer. Math. Soc. 17 (1966), 906-914.

8. - An integral representation of a normal functional on a von Neumann algebra, Trans. Amer. Math. Soc. 125 (1966), 32-46.

9. - A spectral decomposition for self-adjoint elements in the maximal GCR ideal of a von Neumann algebra with applications to noncommutative integration theory, Trans. Amer. Math. Soc. 133 (1968), 281-306.

10. - Commutators in properly infinite von Neumann algebra, Trans. Amer. Math. Soc. 139 (1969), 55-73.

11. - Proper values for the elements of the maximal GCR ideal of a von Neumann algebra, (to appear).

12. - Module homomorphisms of a von Neumann algebra into its center, Trans. Amer. Math. Soc. 140 (1969), 183-193. 
13. R. V. Kadison, Irreducible operator algebras, Proc. Nat. Acad. Sci. U.S.A. 43 (1957), 273-276.

14. I. Kaplansky, The structure of certain operator algebras, Trans. Amer. Math. Soc. 70 (1951), 219-255.

15. — A theorem on rings of operators, Pacific J. Math. 1 (1951), 227-232.

16. — Algebras of type I, Ann. of Math. (2) 56 (1952), 460-472.

17. - Modules over operator algebras, Amer. J. Math. 75 (1953), 839-858.

18. J. Kelly and I. Namioka, Linear topological spaces, Van Nostrand, Princeton, N. J., 1963.

19. M. Nakai, Some expectations in AW*-algebras, Proc. Japan Acad. Sci. 34 (1958), 411-416.

20. J. von Neumann, On rings of operators. Reduction theory, Ann. of Math. (2) 50 (1949), 401-485.

21. C. Rickart, General theory of Banach algebras, Van Nostrand, Princeton, N. J., 1960.

22. S. Sakai, Theory of $W^{*}$-algebras (mimeographed notes), Yale Univ., New Haven, Conn., 1962.

23. I. E. Segal, Decomposition of operator algebras. I, II, Mem. Amer. Math. Soc. No. 9 (1951), $67 \mathrm{pp}$. and $66 \mathrm{pp}$.

24. M. Takesaki, On the Hahn-Banach type theorem and the Jordan decomposition of module linear mappings over some operator algebras, Kōdai Math. Sem. Rep. 12 (1960), 1-10.

25. J. Taylor, The Tomita decomposition of rings of operators, Trans. Amer. Math. Soc. 113 (1964), 30-39.

26. M. Tomita, Representation of operator algebras, Math. J. Okayama Univ. 3 (1954), $142-173$.

27. — Spectral theory of operator algebras. I, Math. J. Okayama Univ. 9 (1959), 63-98.

28. H. Widom, Embedding in algebras of type I, Duke Math. J. 23 (1956), 309-324.

ILLINOIS INSTITUTE OF TeChNOLOGY, Chicago, Illinois 